The Multi-Reference Constant Denominator Perturbation Theory for One Particle Systems and Its Application to the Anharmonic Oscillator
by
(c) Zhida Lan

\author{
A thesis \\ presented to the University of Manitoba \\ in fulfillment of the \\ requirements for the degree of \\ Master of Science \\ in \\ Chemistry
}

Winnipeg, Manitoba

1988 
Permission has been granted to the National Library of Canada to microfilm this thesis and to lend or sell copies of the film.

The author (copyright owner) has reserved other publication rights, and neither the thesis nor extensive extracts from it may be printed or otherwise reproduced without his/her written permission.
L'autorisation a été accordée à la Bibliotheque nationale du Canada de microfilmer cette thèse et de prêter ou de vendre des exemplaires du film.

L'auteur (titulaire du droit d'auteur) se réserve les autres droits de publication; ni la thèse ni de longs extraits de celle-ci ne doivent être imprimés ou autrement reproduits sans son autorisation écrite.

$$
\text { I SBN } \quad 0-315-51615-1
$$




\section{THE MULTI-REFERENCE CONSTANT DENOMINATOR PERTURBATION THEORY FOR ONE PARTICLE SYSTEMS AND ITS APPLICATION TO THE ANHARMONIC OSCILLATOR}

BY

ZHIDA LAN

A thesis submitted to the Faculty of Graduate Studies of the University of Manitoba in partial fulfillment of the requirements of the degree of

MASTER OF SCIENCE

() 1988

Permission has been granted to the LIBRARY OF THE UNIVER-

SITY OF MANITOBA to lend or sell copies of this thesis. to the NATIONAL LIBRARY OF CANADA to microfilm this thesis and to lend or sell copies of the film, and UNIVERSITY MICROFILMS to publish an abstract of this thesis.

The author reserves other publication rights, and neither the thesis nor extensive extracts from it may be printed or otherwise reproduced without the author's written permission. 


\begin{abstract}
In this thesis a multi-reference constant denominator perturbation theory (CDPT) is developed to reduce incomplete basis set errors arising when solving the schrödinger equation with a finite basis set.

The advantage of this method is that very few basis functions are needed and all calculations if carried out to high enough order in the perturbation treatment effectively use a complete basis set. As a first step the theory has been restricted to one particle Hamiltonians and applied to the anharmonic oscillator to study the convergence properties. For perturbation calculations carried out to fifth order results from Pade approximants show an improvement in accuracy of between one and three orders of magnitude.
\end{abstract}


Table of Contents

Abstract I I

Table of contents II

Chapter 1 Review 1

1.0 Introduction 1

1.1 Numerical Methods 2

1.2 Quantum Monte Carlo Methods 3

1.3 Constant Denominator Perturbation Theory 5

1.4 The Anharmonic Oscillator 8

Chapter 2 Theory 11

2.0 Introduction 11

2.1 Peturbation Theory and the $[n, n-1]$ Pade Approximants to Fifth order $\quad 12$

2.2 Multi-reference Constant Denominator Perturbation $\begin{array}{ll}\text { Theory } & 16\end{array}$

2.3 Variational Optimization of the Constant Denominator

2.4 The Anharmonic Oscillator 25

Chapter 3 Results 29

3.0 Introduction 29

3.1 The Effects of the Size and Type of Perturbation on the Convergence 29

A.Variational Results 29

B.Pade Approximant, Results 35

C.General Results 38

D. The Effect of Perturbation Order $\quad 41$ 


\section{Table of Contents (continued)}

3.2 The Effect of starting Point

Acknowledgement

Appendix 1 The Deduction of the Eifth Order Energy

Correction and Variational Energy

Appendix 2 Eigenstate Generating Program

Appendix 3 CDPT Program in MAPLE

A. Introduction

B.hop Subroutine

C.Integral Program intt 1

D. The Program for Multi-reference CDPT to Fifth Order

E.Double Pade Program in MAPLE

F. The Program for Multi-reference CDPT to Third Order

References

List of Tables

Table 1.1 Hartree Fock and Experimental Energies of Hydrogenation

Table 1.2 Comparison of the Molecular Ground State Energies from Exact Type QMC and Experiment

Table $3.1 \mathrm{a}$ The Energy Corrections of CDPT for the Anharmonic Oscillator Having a symmetric Potential 


\section{Table of contents (continued)}

Table 3.1b The Energy Corrections of CDPT for the Anharmonic Oscillator Having a Asymmetric Potential

Table 3.2 The Perturbation Energy Corrections with Different Average Energy $\Delta \mathrm{e}$ and the Results after Pade

Table 3.3a The Energy of Anharmonic Oscillator (1.4.1) 39

Table $3.3 \mathrm{~b}$ The Comparison of Perturbation Energies at the Third order

Table 3.4 The Comparison of Perturbation Energies between Third Order and Fifth order in Case $1=1.0, \mathrm{~m}=1.0$

Table 3.5 The Effect of Starting Point on Ground State

List of Figures

Figure 3.1 Anharmonic Oscillator Potentials 
1.0 Introduction

Since most schrödinger equations cannot be solved analytically, many approaches have been developed to get approximate eigenfunctions and eigenvalues. Almost all these approaches use basis sets which for practical calculations must be finite and therefore incomplete. Sometimes the incompleteness of the basis sets makes the results calculated unreliable. For example, in Table 1.1, hydrogenation energies of four reactions are given. Here results are very dependent on the basis set. Consider, the third reaction

$$
\mathrm{F}_{2}+\mathrm{H}_{2}=2 \mathrm{HF}
$$

results range from -29 to $-134 \mathrm{kcal} \mathrm{mol}^{-1}$.

Table 1.1 Hartree Fock and experimental energies of hydrogenation (kcal/mol)

\begin{tabular}{|c|c|c|c|c|c|c|}
\hline Basis set & $\mathrm{STO}-3 \mathrm{G}$ & $3-21 G$ & $3-21 G^{\star}$ & $6-21 G^{\star}$ & $6-31 G^{\star \star}$ & Expt. \\
\hline $\mathrm{LiF}+\mathrm{H}_{2}$ & & & & & & \\
\hline $\begin{array}{l}=\mathrm{LiH}+\mathrm{HF} \\
\mathrm{CH}_{3} \mathrm{CH}_{2}+\mathrm{H}_{2}\end{array}$ & 31 & 53 & 53 & 49 & 46 & 48 \\
\hline$=2 \mathrm{CH}_{4}$ & -19 & -25 & -25 & -22 & -21 & -19 \\
\hline $\begin{array}{l}\mathrm{E}_{2}+\mathrm{H}_{2} \\
=2 \mathrm{HE}\end{array}$ & -29 & -98 & -98 & -126 & -134 & -133 \\
\hline $\begin{array}{l}\mathrm{NaF}+\mathrm{H}_{2} \\
=\mathrm{HF}+\mathrm{NaH}\end{array}$ & 37 & 30 & 46 & 33 & 30 & 35 \\
\hline
\end{tabular}

Note: This table is from ref. 1 
The question of how this error introduced by the incompleteness of the basis set can be removed or reduced is one of the basic problems in quantum mechanics and quantum chemistry.

\section{I Numerical Methods}

One direct answer to the question above is to generate the wave function and energy numerically[2-5] without recourse to any basis set. In this approach a time independent Schrödinger equation is used

$$
\left[\nabla^{2}+\left(2 m / h^{2}\right)(E-V(R)] \Phi(R)=0\right.
$$

and the whole space is divided into a grid. (e.g. cartesian grid with equidistant points, For practical calculations there are two popular methods. The first is the numerical matrix method $[2,3]$ in which $\nabla^{2} \Phi(R)$ is approximated by the linear combination of wave function values on the near grid points. Then the numerical representation of (1.1.1) becomes many homogeneous linear equations. The problem can be solved after calculating the eigenvalues and eigenvectors of a matrix. The second class of methods, called shooting algorithms [4-9], numerically integrate the schrödinger equation above. Starting energies (or trial functions) are used to get wave function values (or energies). This procedure is iterated and usually converges to the exact (within a given numerical error) eigenvalues and 
eigenfunction values. A good example is the Numerov method ${ }^{[4]}$ which can exactly solve the eigen equation in one dimension case.

In quantum chemistry numerical methods have been used to solve the Hartree Fock equation for atomic, diatomic and linear triatomic systems ${ }^{[6,7]}$. However, for polyatomic molecules, the huge multidimensional space needed due to the representation of wave functions by their grid point values makes these methods impractical. In addition, the coulomb singularities which arise are difficult to treat. [9]

\subsection{Quantum Monte Carlo Methods}

Another class of methods which do not use basis sets is Quantum Monte Carlo (QMC) ${ }^{[10-15]}$. In variational QMC [10-12] the expectation value of energy $\left\langle\mathrm{E}>=\int\left[\mathrm{H} \psi_{\mathrm{T}} / \psi_{\mathrm{T}}\right]\left|\psi_{\mathrm{T}}\right|^{2} \mathrm{~d} \tau / \int\left|\psi_{\mathrm{T}}\right|^{2} \mathrm{~d} \tau\right.$ can be calculated by sampling $\left|\psi_{\mathrm{T}}\right|^{2}$. Trial function $\psi_{\mathrm{T}}$ and initial coordinates of particles are chosen before the calculation. Each particle is then moved randomly one after another to a new position uniformly distributed inside the system. That move is only accepted if the magnitude of $\left|\psi_{T}\right|^{2}$ has increased at the new position compared with the old position. If the move is rejected the configuration is returned to its original state. So after $\mathrm{N}$ steps of random walk

$$
\left\langle E>=(1 / N) \sum_{i}^{N} H \psi_{T}\left(R_{i}\right) / \psi_{T}\left(R_{i}\right)\right.
$$

Because the expectation energy is dependent on $\psi_{T^{\prime}}$ ' this 
approach cannot get back any information missing in the trial function.

In another type of QMC method [13-15], called exact type QMC, the Schrödinger equation is rewritten in imaginary time as

$$
-\partial \psi / \partial t=\left[-D \nabla^{2}+V(R)-E_{t}\right] \psi
$$

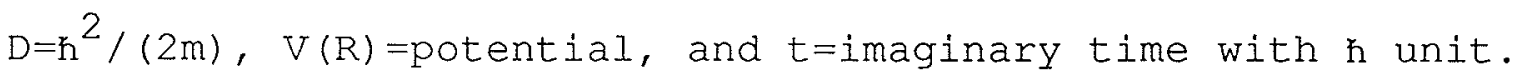

Now in the form of a diffusion equation, various procedures are used to stochastically sample the exact wave function, $\psi(\mathrm{R})$, of the system subject only to statistical error. However, since the wave function generally has nodes, the diffusion process has to be done separately in those regions partitioned by the nodes. The nodes in practical calculations are assumed to be in the same positions as found in the starting trial function $\psi_{\mathrm{T}}(\mathrm{R})$. As shown in Table 1.2 [15] below, the accuracy of the final results are then dependent on the nodal positions used in the calculation. In the case of $\mathrm{H}_{2}$, which has no nodes, its energy calculated is the best result.

Table 1.2 Comparison of the molecular ground state energies from exact type QMC and experiment

\begin{tabular}{lcccc} 
& $\mathrm{H}_{2}$ & $\mathrm{LiH}$ & $\mathrm{Li}_{2}$ & $\mathrm{H}_{2} \mathrm{O}$ \\
QMC & -1.1745 & -8.067 & -14.991 & -76.377 \\
Expt. & -1.17447 & -8.0699 & -14.9967 & -76.4376 \\
\hline
\end{tabular}

Note: The results in table are in atomic units 


\subsection{Constant Denominator Perturbation Theory}

In this thesis a third approach to remove the incomplete basis set error is explored using constant denominator perturbation theory (CDPT). Constant denominator or average energy perturbation theory was first proposed by $A$. Unsöld ${ }^{[16]}$. The main idea behind this method is the following. Consider the first order wave function correction for the i'th state found by Rayleigh Schrödinger perturbation theory

$$
\left|\psi_{1}\right\rangle=\sum_{n \neq i}|n><n| V \mid i>/\left(e_{i}-e_{n}\right)
$$

If $\left|\psi_{1}\right\rangle$ is known the second and third order perturbation energies can be obtained as

$$
E_{2}=\left\langle\psi_{0}\left|V-E_{1}\right| \psi_{1}\right\rangle
$$

and

$$
E_{3}=\left\langle\psi_{1}\left|V-E_{1}\right| \psi_{1}\right\rangle
$$

Here

$$
\begin{aligned}
& \mathrm{E}_{1}=\left\langle\psi_{0}|\mathrm{~V}| \psi_{0}\right\rangle=\langle i|\mathrm{~V}| \mathrm{i}\rangle \\
& \mathrm{V}=\text { perturbation } \\
& \left|\psi_{0}\right\rangle=|i\rangle \text { unperturbed state } \\
& \left\{e_{j}\right\}=\text { the eigenvalues of zero order hamiltonian } \mathrm{H}_{0}
\end{aligned}
$$

Unsöld assumed $e_{i}-e_{n}$ of $E q .(1.3 .1)$ could be replaced by $\Delta e$, the average value of all $e_{i}-e_{n}$. Then an approximate wave function correction is obtained. 


$$
\begin{aligned}
\left|\psi_{1}\right\rangle & \approx(1 / \Delta e) \sum_{n \neq i}|n\rangle\langle n|V| i\rangle \\
& =(1-|i>\langle i|) V \mid i>/ \Delta e \\
& =(1 / \Delta e)\left(1-E_{1}\right) \mid i>
\end{aligned}
$$

This average energy method is usually used to calculate the polarizability $[17-19]$ and estimate the induced dipole moment [20] of molecules. It is also a very popular method to calculate the interaction energy among molecules [21-24]. In these applications one common point is that only the third order perturbation energy of the ground state has been reached. This point is possibly due to the difficulty in evaluating the average energy.

The earliest and easiest way to estimate $\Delta$ e is by replacing $\Delta$ e with empirical quantities, for instance, the first ionization energies ${ }^{[25]}$. It is just this assumption which has given the Unsöld approximation a rather bad reputation, since the final results can be wrong by a factor of 2 . Another possible but time consuming way is to make ab initio calculations of $\Delta e$. Such a non-empirical Unsöld scheme has been proposed by Mulder et al.[25]. Similar to this non-empirical Unsöld method is the generalized Kirkwood method [26-28] in its one parameter version which is actualiy the same approach used by Cullen and zerner ${ }^{[29]}$ in their CDPT method for localized orbitals. The perturbation wave function which is dependent on $\Delta e$ is used as a trial function. For example 


$$
\left|\psi_{\mathrm{T}}\right\rangle=\left|\psi_{0}\right\rangle+\left|\psi_{1}(\Delta \mathrm{e})\right\rangle
$$

Here $\psi_{0}$ and $\psi_{1}$ are the same as those in Eq.(1.3.1)-(1.3.3). $\Delta e$ is then treated as a variational parameter and determined by the Rayleigh-Ritz optimization procedure

$$
W=\left\langle\psi_{\mathrm{T}}|H| \psi_{\mathrm{T}}\right\rangle /\left\langle\psi_{\mathrm{T}} \mid \psi_{\mathrm{T}}\right\rangle \geq \varepsilon_{\text {exact }}
$$

A third non-empirical Unsöld method utilizes the Hylleraas variation principle $[30,31]$ with $\Delta$ e again as a parameter of a trial function. The Hylleraas variation principle is given by

$$
\begin{aligned}
& \left.\mathrm{E}_{2}{ }^{\text {approx }}=2<\psi_{1}^{\text {approx }}\left|\mathrm{V}-\mathrm{E}_{1}\right| \psi_{0}>+<\psi_{1}^{\text {approx }}\left|\mathrm{V}-\mathrm{E}_{1}\right| \psi_{1}^{\text {approx }}\right\rangle \\
& \mathrm{E}_{2} \operatorname{approx}_{\geq \mathrm{E}_{2}}^{\text {exact }}
\end{aligned}
$$

Where $E_{2}$ approx and $E_{2}{ }^{\text {exact }}$ are approximate and exact second order perturbation energies respectively. The inequality $(1.3 .8)$ is only true for the ground state. Taking $\mid \psi_{1}^{\text {approx }}>$ $=(1 / \Delta e)\left(1-E_{1}\right)\left|\psi_{0}\right\rangle$ one can obtain $\Delta$ e by variation in $(1.3 .8)$. In summary, those Unsöld methods above restrict themselves to the ground state because of the use of variation principles which are true for the ground state only. Calculated perturbation energies are to third order and involve parameter $\Delta e$. In this thesis a multi-reference model space is formed and a complete basis is built into a constant denominator perturbation series by using the closure relation. Using a few orthonormal functions as a basis set, one can, not only reduce the incomplete basis error for the 
ground state, but also for excited states. This is demonstrated for the one particle anharmonic oscillator case where the energy perturbation treatment is carried out to fifth order and compared to exact numerical results.

\subsection{The Anharmonic oscillator}

Both in physics and chemistry, the anharmonic oscillator has been an important and interesting model. For example, in quantum statistical mechanics the partition function $Q=\sum$ $\exp \left(-e_{n} / k t\right)$ plays a critical role in the study of the dynamic and thermal properties of a system. Early models for the study of photon dynamics and thermal properties of solids used the harmonic oscillator, since its eigenvalues can be obtained analytically. However a more realistic model is the anharmonic oscillator whose Hamiltonian is given by

$$
H=(1 / 2) p^{2}+k x^{2}+1 x^{3}+m x^{4}
$$

This model has been applied in the field of solid state physics ${ }^{[32]}$, kinetic mechanisms[33,34], thermal dynamics [35] and spectrum theory $[36,37]$. Although the corresponding eigen equation of the anharmonic oscillator (1.4.1) cannot be solved analytically, numerical methods such as the Numerov method $^{[5]}$ can give precise results.

In addition, there have been many non numerical studies of (1.4.1). These include applications of the semiclassical WKB method [38], variational calculations with large basis sets [39], and the employment of specialized basis function expansions $[40,41]$ 


$$
\Phi_{n} \cong \sum_{i} a_{i} \phi_{i}(x)
$$

which when substituted into the schrödinger equation result in a set of linear homogeneous equations for the coefficients $a_{1}$. This last method is however restricted to small $l$ and $\mathrm{m}$.

For normal Rayleigh schrödinger perturbation treatments, i.e. perturbation $V=1 x^{3}+m x^{4}$ and $H_{0}=(1 / 2) p^{2}+k x^{2}$, it has been pointed out by Bender and $W u^{[42]}$ that the energy perturbation series diverges asymptotically for any $m$. Other possible partitionings of the anharmonic oscillator have been considered by Patnaik[43], and Halliday [44]. For example, in the case of Patnaik's work on the quartic oscillator $(1=0)$, the Hamiltonian is partitioned using a ladder operator technique. The resulting zero order eigenfunction basis was truncated to an arbitrary number of 19 functions. A Rayleigh Schrödinger perturbation treatment of the energy was then carried out to third order. Such a calculation is not simple because of the two steps involved and relatively large basis set used. Even so his final results as will be seen in Chapter 3. are not satisfactory.

Recently Killingbeck $[45,46]$ has applied the hypervirial theorem to the anharmonic oscillator to obtain an energy expansion. The value of this method is that no wave function is required and therefore there is no basis set incompleteness problem. After evaluating the Kilingbeck's energy expansion to the $32^{\prime}$ th order Lai and Madan $[47,48]$ calculated the $[8,8]$ Pade approximants. However, even at this 
very high level of theory, the final results obtained are accurate only to the third decimal for small $l$ and $m$. These results are compared in Chapter 3 with the theory developed in this thesis. 


\section{Chapter 2 Theory}

\subsection{Introduction}

In this Chapter a multi-reference constant denominator perturbation theory is developed for single particle Hamiltonians. In the first step of this theory a basis set of several zero order reference states is selected by a variational calculation with the Hamiltonian. Once this model space has been constructed a perturbation treatment which uses the closure condition to complete the basis is then carried out separately for each of these reference states. The wave function and energy corrections obtained, however, are dependent upon the constant denominator $\Delta \mathrm{e}$ used in the calculation. This $\Delta e$ can be optimally chosen using an variational method, which gives an exact upper bound for the ground state and approximate ones for the excited states. Alternatively, Pade approximants can also be used to obtain results for the energy which are relatively independent of the $\Delta$ e values.

We begin by reviewing perturbation theory and its relationship to the $[n, n-1]$ Pade approximants (Section 2.1). This is followed by the development of the multi-reference constant denominator perturbation theory for one particle Hamiltonians (Section 2.2). The method for the variational optimization of the constant denominator is then derived (Section 2.3). Finally for the application to anharmonic oscillator details of the construction of the model reference 
space are outlined (Section 2.4).

2.1 Perturbation Theory and the $[n, n-1]$ Pade Approximants to Fifth Order

Suppose we want to solve the eigenvalue problem

$$
H\left|\Phi_{i}\right\rangle=\left(H_{0}+V\right)\left|\Phi_{i}\right\rangle=\varepsilon_{i}\left|\Phi_{i}\right\rangle
$$

where we know the eigenfunctions and eigenvalues of $\mathrm{H}_{0}$.

$$
\mathrm{H}_{0}\left|\phi_{i}\right\rangle=e_{i}\left|\phi_{i}\right\rangle \quad \text { or } \quad \mathrm{H}_{0}|i\rangle=e_{i}|i\rangle
$$

If projection operators $P^{\prime}$ and $Q^{\prime}$ are defined as

$$
\begin{aligned}
& P^{\prime}=|i><i| \\
& Q^{\prime}=\sum_{n \neq i}|n><n|
\end{aligned}
$$

The wave function $\left|\Phi_{i}\right\rangle$ and Schrödinger equation can be rewritten as

$$
\begin{gathered}
\left.\left|\Phi_{i}\right\rangle=\left|\phi_{i}>+Q^{\prime}\right| \Phi_{i}\right\rangle \\
\left(\xi-H_{0}\right)\left|\Phi_{i}>=\left(\xi-\varepsilon_{i}+V\right)\right| \Phi_{i}>
\end{gathered}
$$

where $\xi$ is an arbitrary constant.

Substitution of (2.1.4) into (2.1.5) followed by iteration with 


$$
\begin{gathered}
\left|\Phi_{i}\right\rangle=\mid \phi_{i}>\text { as an initial guess yields } \\
\left|\Phi_{i}\right\rangle=\sum_{q=0}^{\infty} \mid \psi_{q}> \\
\left.\left|\psi_{q}>=\left\{R\left(\xi-\varepsilon_{i}+V\right)\right\}^{q}\right| \phi_{i}\right\rangle
\end{gathered}
$$

where $R$, the resolvent is given by

$$
R=Q^{\prime}\left(\xi-H_{0}\right)^{-1} Q^{\prime}
$$

The corresponding energy is obtained through the use of the intermediate normalization condition $\left\langle\Phi_{i} \mid \phi_{i}\right\rangle=1$ with

$$
\begin{aligned}
& \varepsilon_{i}=\left\langle\phi_{i}|H| \Phi_{i}>=\sum_{q=0}^{\infty} E^{q}\right. \\
& E_{q+1}=\left\langle\phi_{i}\left|V\left\{R\left(\xi-\varepsilon_{i}+V\right)\right\}{ }^{q}\right| \phi_{i}\right\rangle
\end{aligned}
$$

For Brillouin Wigner perturbation theory $\xi$ is set to $\varepsilon_{i}$, and the resulting expressions for wave function to second order and energy to fifth order become

$$
\begin{aligned}
\mid \psi> & =\sum_{q=0}^{2}\left|\psi_{q}\right\rangle \\
\mid \psi_{q}>= & {\left[\sum_{n \neq i}\left(1 /\left(e_{i}-e_{n}\right)\right)|n>\langle n| v]{ }^{q}\left|\phi_{i}\right\rangle\right.} \\
\varepsilon_{i} & =\sum_{q=0}^{5} E_{q} \\
E_{q+1} & =\left\langle\phi_{i}\right| V\left[\sum_{n \neq i}\left(1 /\left(e_{i}-e_{n}\right)\right)|n>\langle n| V]^{q}\left|\phi_{i}\right\rangle\right.
\end{aligned}
$$

In the case of Rayleigh Schrödinger perturbation theory $\xi$ is set to $e_{0}$. The first and second wave function corrections 
and corresponding perturbation energies to fifth order for state $\mid$ i> are then given as

$$
\begin{aligned}
& \left|\psi_{1}\right\rangle=\sum_{n \neq i}|n\rangle\langle n|V| i\rangle /\left(e_{i}-e_{n}\right) \\
& \left|\psi_{2}\right\rangle=\sum_{n \neq i}|n\rangle\left\langle n|V| \psi_{1}\right\rangle /\left(e_{i}-e_{n}\right) \quad-E_{1} \sum_{n \neq i}|n\rangle\left\langle n \mid \psi_{1}\right\rangle /\left(e_{i}-e_{n}\right) \\
& E_{1}=\langle i|V| i\rangle \\
& E_{2}=\left\langle i|V| \psi_{1}\right\rangle \\
& E_{3}=\left\langle\psi_{1}\left|V-E_{1}\right| \psi_{1}\right\rangle \\
& E_{4}=\left\langle\psi_{1}\left|V-E_{1}\right| \psi_{2}\right\rangle-E_{2}\left\langle\psi_{1} \mid \psi_{1}\right\rangle \\
& E_{5}=\left\langle\psi_{2}\left|V-E_{1}\right| \psi_{2}>-E_{3}\left\langle\psi_{1}\left|\psi_{1}>-E_{2}<\psi_{1}\right| \psi_{2}\right\rangle-E_{2}\left\langle\psi_{2} \mid \psi_{1}\right\rangle\right.
\end{aligned}
$$

Now consider the trial function

$$
\left.\left|\psi_{\mathrm{T}}\right\rangle=\left|\phi_{0}>+\mathrm{c}_{1}\right| \psi_{1}\right\rangle+\mathrm{c}_{2}\left|\psi_{2}\right\rangle
$$

Following the Rayleigh Ritz variational principle

$$
\begin{aligned}
& \mathrm{W}=\left\langle\psi_{\mathrm{T}}|\mathrm{H}| \psi_{\mathrm{T}}>/\left\langle\psi_{\mathrm{T}} \mid \psi_{\mathrm{T}}\right\rangle \geq \varepsilon_{0}\right. \\
& w\left(c_{1}, c_{2}\right)=e_{0}+e_{1}+2 \sum_{p=1}^{2} c_{p} e_{p+1} \underset{p q=1}{2}<\psi_{p}\left|\xi^{\prime}-H_{0}\right| \psi_{q}>c_{p} c_{q} \\
& \underset{p q=1}{+\sum_{p}<\psi}|V| \psi_{q}>C_{p} C_{q}+\Delta W \sum_{p q=1}^{2}<\psi_{p} \mid \psi_{q}>C_{p} c_{q}
\end{aligned}
$$


without the last term, i.e.

$$
\begin{aligned}
w_{2}\left(c_{1}, c_{2}\right)= & e_{0}+e_{1}+e_{2}\left(2 c_{1}-c_{2}^{2}\right)+e_{3}\left(c_{1}^{2}+2 c_{2}-2 c_{1} c_{2}\right) \\
& +e_{4}\left(2 c_{1} c_{2}-c_{2}^{2}\right)+c_{2}^{2} e_{5}
\end{aligned}
$$

where generally $\mathrm{w}_{2}\left(c_{1}, c_{2}\right) \geq w\left(c_{1}, c_{2}\right)$

If $c_{2}=0$, the variation of $(2.1 .24)$ produces what is known as the $[1,0]$ Pade, that is

$$
\begin{aligned}
E[1,0] & =w_{2}\left(c_{1}^{\prime}, 0\right)=e_{0}+e_{1}+c_{1}^{\prime} e_{2} \\
\text { With } \quad c_{1}^{\prime} & =1 /\left(1-e_{3} / e_{2}\right)
\end{aligned}
$$

Similarly if $c_{1} \neq 0$ and $c_{2} \neq 0$, the variation of $(2.1 .24)$ produces the $[2,1]$ Pade.

$$
\begin{aligned}
& \mathrm{E}[2,1]=\mathrm{W}_{2}\left(\mathrm{c}_{1}^{*}, \mathrm{c}_{2}^{*}\right)=e_{0}+e_{1}+\mathrm{c}_{1}^{*} e_{2}+\mathrm{c}_{2}^{*} e_{3} \\
& \text { With } c_{1}^{*}=\left[e_{2}\left(e_{4}-e_{5}\right)-e_{3}\left(e_{3}-e_{4}\right)\right] /\left[\left(e_{2}-e_{3}\right)\left(e_{4}-e_{5}\right)-\left(e_{3}-e_{4}\right)^{2}\right] \\
& \text { and } c_{2}^{*}=\left[e_{3}\left(e_{2}-e_{3}\right)-e_{2}\left(e_{3}-e_{4}\right)\right] /\left[\left(e_{2}-e_{3}\right)\left(e_{4}-e_{5}\right)-\left(e_{3}-e_{4}\right)^{2}\right]
\end{aligned}
$$

Since the $[1,0]$ and $[2,1]$ Pade are usually upper bounds on the perturbation expansion one can define a new energy series based on these Pades which will be generally well behaved

$$
\begin{aligned}
& e_{0}^{*}+e_{1}^{*}=e_{0}+e_{1} \\
& e_{2}^{*}=w_{2}\left(c_{1}^{\prime}, 0\right)-\left(e_{0}+e_{1}\right)
\end{aligned}
$$




$$
e_{3}^{*}=w_{2}\left(c_{1}^{*}, c_{2}^{*}\right)-w_{2}\left(c_{1}^{\prime}, 0\right)
$$

If we once again apply the Pade approximant method on this series at the $[1,0]$ level we obtain

$$
w[1,0]=e_{0}^{*}+e_{1}^{*}+e_{2}^{*} /\left(1-e_{3}^{*} / e_{2}^{*}\right)
$$

This procedure will be called the double Pade ${ }^{[49]}$.

\subsection{Multi-Reference Constant Denominator Perturbation Theory} For One Particle Hamiltonians

The first step in multi-reference theory is to define an incomplete basis set called the model space $(\mid i>; i=0, \ldots, M)$. This basis set satisfies the conditions

$$
\begin{aligned}
& \langle i \mid j\rangle=\delta_{i j} \\
& \left\langle i|H| j>=\delta_{i j} e_{i}\right.
\end{aligned}
$$

where $\mathrm{H}$ is the Hamiltonian of the one particle system. Projection operators are then defined by

$$
\begin{aligned}
& \mathrm{P}=\sum_{i=0}^{M}|i><i| \\
& Q=\sum_{i=0}^{M}|i><i| \text { or } Q=\sum_{j=M+1}|j><j|
\end{aligned}
$$

where 1 is unit operator and states $|j\rangle$ form an orthonormal complement to the model space. In order to avoid confusion later on, the difference between these projection operators and the primed ones given in equations (2.1.2) and (2.1.3) of 
Section 2.1 should be carefully noted.

Following the constant denominator approach [29], states outside the model space have the average energy $e^{*}$. This results in a zero order Hamiltonian of the form

$$
\mathrm{H}_{0}=\sum_{i=0}^{M} e_{i}|i><i|+e^{\star} Q
$$

The corresponding perturbation operator is then

$$
\begin{aligned}
V & =H-H_{0}=H-\left(\sum_{i=0}^{M} e_{i}|i><i|+e^{*} Q\right) \\
& =H-e^{*}-\sum_{i=0}^{M}\left(e_{i}-e^{*}\right)|i><i|
\end{aligned}
$$

The resulting Rayleigh Schrödinger perturbation wave function corrections to second order and corresponding energies to fifth order are given in eq.(2.1.15)-(2.1.21) of Section 2.1. In order to solve these equations consider the perturbation acting on an intermediate state $|n\rangle(n \leq M)$ in the model space.

$$
\begin{aligned}
V \mid n> & =\left[\underset{j=0}{M} e_{j}|j\rangle\langle j|-e^{*} Q\right] \mid n> \\
& =\left(H-e_{n}\right) \mid n>
\end{aligned}
$$

where the properties $\langle i \mid j\rangle=\delta_{i j}$ and $Q|n\rangle=0$ have been used. Hence

$$
E_{1}=\langle i|V| i\rangle=\left\langle i\left|H-e_{i}\right| i\right\rangle=0
$$

Now let $Q$ act on $\mathrm{V} \mid \mathrm{n}>$

$$
\begin{aligned}
Q V \mid n> & =Q\left(H-e_{n}\right) \mid n> \\
& =(1-P) H \mid n>
\end{aligned}
$$




$$
\begin{aligned}
\mathrm{QV}|\mathrm{n}\rangle & =\left(\mathrm{H}-\sum_{j=0}^{\mathrm{M}}|j\rangle\langle j| \mathrm{H}\right)|\mathrm{n}\rangle \\
& =\left(\mathrm{H}-\mathrm{e}_{\mathrm{n}}\right)|\mathrm{n}\rangle
\end{aligned}
$$

Hence $Q V|n>=V| n>$

Now the first order wave function correction $\left|\psi_{1}\right\rangle$ can be obtained as

$$
\begin{aligned}
\left|\psi_{1}\right\rangle & =\sum_{n \neq i}|n\rangle\langle n|V| i\rangle /\left(e_{i}-e_{n}\right) \\
& =\sum_{n \neq i}^{M}|n\rangle\left\langle n|V| i>/\left(e_{i}-e_{n}\right)+\left(1 /\left(e_{i}-e^{*}\right)\right) Q V \mid i\right\rangle
\end{aligned}
$$

Defining $\Delta e=e_{i}-e^{*}$ and using the relation

$$
\langle\mathrm{n}|\mathrm{V}| \mathrm{i}\rangle=\left\langle\mathrm{n}\left|\mathrm{H}-\mathrm{e}_{\mathrm{i}}\right| \mathrm{i}\right\rangle=0
$$

equation (2.1.11) finally becomes

$$
\left|\psi_{1}\right\rangle=(1 / \Delta e)\left(H-e_{i}\right)|i\rangle=(1 / \Delta e) V \mid i>
$$

In order to solve for the corresponding second and third order energy corrections, equations (2.1.18) and (2.1.19), consider $V \mid \psi_{1}>$ or $(1 / \Delta e) V^{2} \mid i>$

$$
\begin{aligned}
& \mathrm{V}^{2}=\left(\underset{j=0}{M} \sum_{j} \mid j>\langle j|-e^{*} Q\right)\left(H-\sum_{j=0}^{M} e_{j} \mid j>\langle j|-e^{*} Q\right) \\
& =H^{2}-\sum_{j=0}^{M} e_{j} H|j\rangle\left\langle j\left|-e^{\star} H Q-\sum_{j=0}^{M} e_{j}\right| j\right\rangle\left\langle j\left|H+\sum_{j=0}^{M} e_{j}^{2}\right| j\right\rangle\langle j|-e^{*} Q H+e^{*}{ }^{2} Q \\
& V^{2}\left|i>=H^{2}\right| i>-\sum_{j=0}^{M} e_{j} H|j\rangle\left\langle j\left|i>-e^{*} H Q\right| i>-\sum_{j=0}^{M} e_{j}\right| j>\langle j|H| i>+ \\
& +\sum_{j=0}^{M} e_{j}^{2}|j\rangle\left\langle j\left|i>-e^{*} \mathrm{QH}\right| i>+e^{*} \stackrel{2}{Q}\right| i> \\
& =\left(\mathrm{H}^{2}-e_{i} \mathrm{H}-e_{i}^{2}+e_{i}^{2}-e^{*} \mathrm{QH}\right)|i\rangle
\end{aligned}
$$




$$
=\left(H^{2}-e_{i} H-e^{*} H+e^{*} e_{i}\right)|i\rangle
$$

Therefore $\quad V\left|\psi_{1}\right\rangle=\left[H-e_{i}+(1 / \Delta e)\left(H^{2}-2 e_{i} H+e_{i}^{2}\right)\right] \mid i>$

$$
=\left(\left(H-e_{i}\right) / \Delta e\right)\left(H-e_{i}+\Delta e\right) \mid i>
$$

The resulting energy expressions for the second and third order corrections are then

$$
\begin{aligned}
\mathrm{E}_{2} & =\left\langle i|V| \psi_{1}\right\rangle=(1 / \Delta e)\left\langle i\left|\left(H-e_{i}\right)^{2}\right| i\right\rangle \\
E_{3} & =\left\langle\psi_{1}\left|V-E_{1}\right| \psi_{1}\right\rangle=\left\langle\psi_{1}|V| \psi_{1}\right\rangle \\
& =(1 / \Delta e)\langle i|\left(H-e_{i}\right)\left[\left(H-e_{i}\right)+(1 / \Delta e)\left(H-e_{i}\right)^{2}|i\rangle\right. \\
& =(1 / \Delta e)\left[\left\langle i\left|\left(H-e_{i}\right)^{2}\right| i\right\rangle+(1 / \Delta e)\left\langle i\left|\left(H-e_{i}\right)^{3}\right| i\right\rangle\right]
\end{aligned}
$$

Equation (2.2.16) can also be used to construct the second order wave function, $\left|\psi_{2}\right\rangle$. Decomposing (2.1.16) into its $P$ and Q space components yields

$$
\begin{aligned}
& \left|\psi_{2}>=\sum_{n \neq i}^{M}\right| n><n|V| \psi_{1}>/\left(e_{i}-e_{n}\right)+Q V \mid \psi_{1}>/ \Delta e \\
& =\sum_{n \neq i}^{M}|n\rangle\left\langle n|V| \psi_{1}>/\left(e_{i}-e_{n}\right)+(1 / \Delta e) \underset{n=0}{M}\left(1-\sum_{n=0}|n\rangle\langle n|\right) v \mid \psi_{1}\right\rangle \\
& =\sum_{n \neq i}^{M} \mid n>\left\langle n|V| \psi_{1}>/\left(e_{i}-e_{n}\right)+(1 / \Delta e) V\left|\psi_{1}>-(1 / \Delta e) \sum_{n=0}^{M}\right| n\right\rangle\left\langle n|V| \psi_{1}\right\rangle
\end{aligned}
$$

where we have used relation (2.2.8). If now (2.2.16) is substituted into (2.2.19) the final 
result is

$$
\begin{aligned}
\mid \psi_{2}>= & -\left\langle i\left|\left(H-e_{i}\right)^{2}\right| i>/(\Delta e)^{2}\left|i>+\left(H-e_{i}\right) /(\Delta e)\right| i>+\left[\left(H-e_{i}\right) / \Delta e\right]^{2}\right| i> \\
& \left.+\sum_{n \neq i}|n><n|\left(H-e_{i}\right)^{2} \mid i>\left[1 /\left(e_{i}-e_{n}\right)-1 / \Delta e\right)\right] / \Delta e
\end{aligned}
$$

For the determination of the fourth and fifth order energy corrections let $V$ act on (2.2.16) and (2.2.19).

$$
\begin{aligned}
V^{2}\left|\psi_{1}\right\rangle= & \left(H-e^{*}-\sum_{j=0}^{M}\left(e_{j}-e^{*}\right)|j\rangle\langle j|\right) V\left|\psi_{1}\right\rangle \\
= & \left.\left(H-e_{i}+\Delta e\right) V\left|\psi_{1}>-\sum_{j=0}^{M}\left(e_{j}-e_{i}+\Delta e\right)\right| j\right\rangle\left\langle j|V| \psi_{1}>\right. \\
= & \left(H-e_{i}\right)\left(\Delta e+H-e_{i}\right)^{2} / \Delta e \mid i> \\
& -(1 / \Delta e) \sum_{j=0}^{M}\left(e_{j}-e_{i}+\Delta e\right)|j\rangle\left\langle j\left|\left\langle H-e_{i}\right)^{2}\right| i\right\rangle
\end{aligned}
$$

Similarly

$$
\mathrm{V}\left|\psi_{2}\right\rangle=\sum_{n \neq i}^{M} V \mid n>\left\langle n|V| \psi_{1}>/\left(e_{i}-e_{n}\right)+V^{2} / \Delta e\left|\psi_{1}>-(1 / \Delta e) \sum_{n=0}^{M} V\right| n>\left\langle n|V| \psi_{1}\right\rangle\right.
$$

substituting $(2.2 .7),(2.2 .16)$ and $(2.2 .21)$ results in

$$
\begin{aligned}
V\left|\psi_{2}\right\rangle= & \sum_{n \neq i}^{M}\left(H-e_{n}\right)|n\rangle\left\langle n|V| \psi_{1}>/\left(e_{i}-e_{n}\right)+V^{2} / \Delta e\right| \psi_{1}> \\
& -(1 / \Delta e) \sum_{n=0}^{M}\left(H-e_{n}\right) \mid n>\langle n|V| \psi\rangle \\
= & \sum_{n \neq i}^{M}\left(H-e_{n}\right) \mid n>\left\langle n\left|\left(H-e_{i}\right)^{2}\right| i>/\left[\left(e_{i}-e_{n}\right) \Delta e\right]\right. \\
& -1 /(\Delta e)^{2} \sum_{n=0}^{M}\left(H-e_{i}+\Delta e\right) \mid n>\left\langle n\left|\left(H-e_{i}\right)^{2}\right| i>\right. \\
& +1 /(\Delta e)^{2}\left(H-e_{i}\right)\left(H-e_{i}+\Delta e\right)^{2} \mid i>
\end{aligned}
$$


In the case of the fourth order energy, the two terms in equation (2.1.20) become

$$
\begin{aligned}
& \left\langle\psi_{1}\left|V-E_{1}\right| \psi_{2}\right\rangle=\left\langle\psi_{1}|V| \psi_{2}\right\rangle \\
& =(1 / \Delta e)^{3}\left[\sum_{n \neq i}^{M}<i\left|\left(H-e_{i}\right)\left(H-e_{n}\right)\right| n><n\left|\left(H-e_{i}\right)^{2}\right| i>\Delta e /\left(e_{i}-e_{n}\right)\right. \\
& -\sum_{n=0}^{M}<i\left|\left(H-e_{i}\right)\left(H-e_{n}\right)\right| n>\left\langle n\left|\left(H-e_{i}\right)^{2}\right| i>+<i\left|\left(H-e_{i}\right)^{2}\left(H-e_{i}+\Delta e\right)^{2}\right| i>\right. \\
& \left.-\sum_{n=0}^{M}<i\left|\left(H-e_{i}\right)\left(e_{n}-e_{i}+\Delta e\right)\right| n><n\left|\left(H-e_{i}\right)^{2}\right| i>\right] \\
& =(1 / \Delta e)^{3} \sum_{n \neq i}^{M}|<i|\left(H-e_{i}\right)^{2}|n>|^{2} \Delta e /\left(e_{i}-e_{n}\right)-\sum_{n \neq i}^{M}|<i|\left(H-e_{i}\right)^{2}|n>|^{2} \\
& \left.-<i\left|\left(H-e_{i}\right)^{2}\right| i\right\rangle^{2}+<i\left|\left(H-e_{i}\right)^{4}\right| i>+2 \Delta e<i\left|\left(H-e_{i}\right)^{3}\right| i> \\
& +(\Delta e)^{2}<i \mid\left(H-e_{i}\right)^{2}[i>] \\
& E_{2}<\psi_{1} \mid \psi_{1}>=(1 / \Delta e)^{3}\left[\left\langle i\left|\left(H-e_{i}\right)^{2}\right| i>\right]^{2}\right.
\end{aligned}
$$

Therefore

$$
\begin{aligned}
E_{4}= & \left\langle\psi_{1}\left|V-E_{1}\right| \psi_{2}\right\rangle-E_{2}\left\langle\psi_{1} \mid \psi_{1}\right\rangle \\
= & (1 / \Delta e)^{3} \sum_{n \neq i}^{M} \mid\left\langle i\left|\left(H-e_{i}\right)^{2}\right| n>\left.\right|^{2}\left(\Delta e /\left(e_{i}-e_{n}\right)-1\right)-2\left\langle i\left|\left(H-e_{i}\right)^{2}\right| i\right\rangle^{2}\right. \\
& +\left\langle i\left|\left(H-e_{i}\right)^{4}\right| i>+2 \Delta e<i\left|\left(H-e_{i}\right)^{3}\right| i>+(\Delta e)^{2}\left\langle i\left|\left(H-e_{i}\right)^{2}\right| i\right\rangle\right]
\end{aligned}
$$

Similarly in the case of the fifth order energy, the four terms found in equation (2.1.21) are given by the following expressions.

$$
\begin{aligned}
E_{2}<\psi_{1} \mid \psi_{2}> & =E_{2}\left[\left\langle i\left|\left(H-e_{i}\right)^{2}\right| i>+<i\left|\left(H-e_{i}\right)^{3}\right| i>/ \Delta e\right] /(\Delta e)^{2}\right. \\
& \left.=\left[<i\left|\left(H-e_{i}\right)^{2}\right| i\right\rangle^{2}+<i\left|\left(H-e_{i}\right)^{3}\right| i><i\left|\left(H-e_{i}\right)^{2}\right| i>/ \Delta e\right] /(\Delta e)^{3}
\end{aligned}
$$


$E_{3}\left\langle\psi_{1}\right| \psi_{1}>=\left[\left\langle i\left|\left(H-e_{i}\right)^{2}\right| i\right\rangle^{2}+\left\langle i\left|\left(H-e_{i}\right)^{3}\right| i>\left\langle i\left|\left(H-e_{i}\right)^{2}\right| i>/ \Delta e\right] /(\Delta e)^{3}\right.\right.$

and

$$
\begin{aligned}
& \left\langle\psi_{2}\left|\mathrm{~V}-\mathrm{E}_{1}\right| \psi_{2}\right\rangle=\left\langle\psi_{2}|\mathrm{~V}| \psi_{2}\right\rangle \\
& =-\left(2 /(\Delta e)^{4}\right)<i\left|\left(H-e_{i}\right)^{2}\right| i><i\left|\left(H-e_{i}\right)^{3}\right| i>-3<i\left|\left(H-e_{i}\right)^{2}\right| i>^{2} /(\Delta e)^{3} \\
& +(1 / \Delta e) \sum_{n \neq i}^{2}\left\{|<i|\left(H-e_{i}\right)^{2}|n>|^{2}\left[1 /\left(e_{i}-e_{n}\right)-1 / \Delta e\right]\left[2+2\left(e_{i}-e_{n}\right) / \Delta e\right]\right. \\
& +2\left[1 /\left(e_{i}-e_{n}\right)-1 / \Delta e\right]<i\left|\left(H-e_{i}\right)^{3}\right| n><n\left|\left(H-e_{i}\right)^{2}\right| i>/ \Delta e \\
& \left.-\left(e_{n}-e_{i}+\Delta e\right)|<i|\left(H-e_{i}\right)^{2}|n>|^{2} /(\Delta e)^{2}\right\}+3<i\left|\left(H-e_{i}\right)^{4}\right| i>/(\Delta e)^{3} \\
& +3<i\left|\left(H-e_{i}\right)^{3}\right| i>/(\Delta e)^{2}+<i\left|\left(H-e_{i}\right)^{2}\right| i>/ \Delta e+<i\left|\left(H-e_{i}\right)^{5}\right| i>/(\Delta e)^{4}
\end{aligned}
$$

Details of the rather lengthy derivation of this last term (2.2.29) are given in Appendix 1A. The resulting fifth order energy is then

$$
\begin{aligned}
E_{5}= & <\psi_{2}\left|V-E_{1}\right| \psi_{2}>-E_{3}<\psi_{1}\left|\psi_{1}>-2 E_{2}<\psi_{1}\right| \psi_{2}> \\
= & -5<i\left|\left(H-e_{i}\right)^{2}\right| i><i\left|\left(H-e_{i}\right)^{3}\right| i>/(\Delta e)^{4}-6<i\left|\left(H-e_{i}\right)^{2}\right| i>^{2} /(\Delta e)^{3} \\
& +(1 / \Delta e)^{2} \sum_{n \neq i}^{M}\left\{|<i|\left(H-e_{i}\right)^{2}|n>|^{2}\left[1 /\left(e_{i}-e_{n}\right)-1 / \Delta e\right]\left[2+2\left(e_{i}-e_{n}\right) / \Delta e\right]\right. \\
& +2\left[1 /\left(e_{i}-e_{n}\right)-1 / \Delta e\right]<i\left|\left(H-e_{i}\right)^{3}\right| n><n\left|\left(H-e_{i}\right)^{2}\right| i>/ \Delta e \\
& \left.-\left(e_{n}-e_{i}+\Delta e\right)|<i|\left(H-e_{i}\right)^{2}|n>|^{2} /(\Delta e)^{2}\right\}+3<i\left|\left(H-e_{i}\right)^{4}\right| i>/(\Delta e)^{3} \\
& +3<i\left|\left(H-e_{i}\right)^{3}\right| i>/(\Delta e)^{2}+<i\left|\left(H-e_{i}\right)^{2}\right| i>/ \Delta e+<i\left|\left(H-e_{i}\right)^{5}\right| i>/(\Delta e)^{4}
\end{aligned}
$$




\subsection{Variational Optimization of the Constant Denominator}

As pointed out in the introduction the choice of the constant denominator, $\Delta e$, resulting from the partitioning of $\mathrm{H}$ into $\mathrm{H}_{0}$ and $\mathrm{V}$ is rather arbitrary. In this section an approximate variational method is developed for finding an optimal $\Delta e$.

Assume that states $\left|\Phi_{j}\right\rangle(j=0, \ldots, i-1)$ are exact eigenstates of $\mathrm{H}$ with $\left\{\varepsilon_{j}\right\} \quad(j=0, \ldots, i-1)$ being the corresponding eigenvalues. Then the following variational principle is true.

$$
W=\left\langle\psi_{T}\left|\left(H-\sum_{j=0}^{i-1} \varepsilon_{j}\left|\Phi_{j}><\Phi_{j}\right|\right)\right| \psi_{T}>/<\psi_{T}\right| \psi_{T}>\geq \varepsilon_{\text {exact }}
$$

where $\varepsilon_{\text {exact }}$ is the exact eigenvalue of i'th state.

If $|j\rangle$ and $e_{j}(j=0, \ldots, i-1)$ are not far from the exact eigenstates and corresponding eigenvalues. Then $\left|\Phi_{j}\right\rangle$ and $\varepsilon_{j}$ $(j=0, \ldots, i-1)$ in $(2.3 .1)$ can be substituted by $|j\rangle$ and $e_{j}$ to evaluate an approximate $\Delta$ e.

Now choose as the trial function the second order perturbation wave function

$$
\begin{aligned}
& \left|\psi_{\mathrm{T}}\right\rangle=|i\rangle+\left|\psi_{1}\right\rangle+\left|\psi_{2}\right\rangle \\
& =\left[1-(1 / \Delta e)^{2}\left\langle i\left|\left(H-e_{i}\right)^{2}\right| i\right\rangle\right]|i\rangle+2\left(H-e_{i}\right) / \Delta e|i\rangle \\
& +(1 / \Delta e)^{2}\left(H-e_{i}\right)^{2}\left|i>+\sum_{n \neq i}^{M}\right| n><n\left|\left(H-e_{i}\right)^{2}\right| i>\left[1 /\left(e_{i}-e_{n}\right)\right. \\
& -1 / \Delta e] / \Delta e
\end{aligned}
$$

Here the equations $(2.2 .13)$ and $(2.2 .20)$ have been used. The 
corresponding norm, $\left\langle\psi_{\mathrm{T}} \mid \psi_{\mathrm{T}}\right\rangle$ is then given as

$$
\begin{aligned}
\left\langle\psi_{\mathrm{T}} \mid \psi_{\mathrm{T}}\right\rangle= & (1 / \Delta e)^{4}\left[-<i\left|\left(H-e_{i}\right)^{2}\right| i\right\rangle^{2}+<i\left|\left(H-e_{i}\right)^{4}\right| i> \\
& \left.-\sum_{n \neq i}^{M}|<n|\left(H-e_{i}\right)^{2}|i>|^{2}\right]+4<i\left|\left(H-e_{i}\right)^{3}\right| i>/ \Delta e^{3} \\
& +(1 / \Delta e)^{2}\left[4<i\left|\left(H-e_{i}\right)^{2}\right| i>+\sum_{n \neq i}^{M}|<n|\left(H-e_{i}\right)^{2}|i>|^{2}\right. \\
& \left.\times\left(1 /\left(e_{i}-e_{n}\right)^{2}\right)\right]+1
\end{aligned}
$$

Details of the intermediate steps used to obtain equation (2.3.3) can be found in Appendix 1B.

Consider now $\mathrm{H}^{*}\left|\psi_{\mathrm{T}}\right\rangle$ where

$$
H^{*}=H_{j=0}^{i-1} e_{j}|j\rangle\langle j|
$$

Let $\mathrm{H}^{*}$ act on $(2.3 .2)$ and use equations (2.2.1) and (2.2.2)

$$
\begin{aligned}
& \mathrm{H}^{*}\left|\psi_{\mathrm{T}}\right\rangle=e_{i}\left[1-(1 / \Delta \mathrm{e})^{2}\left\langle i\left|\left(H-e_{i}\right)^{2}\right| i>\right] \mid i>\right. \\
& +\left[2 e_{i} / \Delta e+\left(1-\left\langle i\left|\left(H-e_{i}\right)^{2}\right| i>/(\Delta e)^{2}\right)\right]\left(H-e_{i}\right)|i\rangle\right. \\
& +\left[e_{i} /(\Delta e)^{2}+2 / \Delta e\right]\left(H-e_{i}\right)^{2}\left|i>+(1 / \Delta e)^{2}\left(H-e_{i}\right)^{3}\right| i> \\
& +\sum_{n \neq i}^{M}\left(H-e_{i}\right)|n><n|\left(H-e_{i}\right)^{2} \mid i>\left[1 /\left(e_{i}-e_{n}\right)-1 / \Delta e\right] / \Delta e \\
& +e_{i} \sum_{n \neq i}^{M}|n><n|\left(H-e_{i}\right)^{2} \mid i>\left[1 /\left(e_{i}-e_{n}\right)-1 / \Delta e\right] / \Delta e \\
& -(1 / \Delta e) \sum_{j=0}^{i-1} e_{j} \mid j>\left\langle j\left|\left(H-e_{i}\right)^{2}\right| i>/\left(e_{i}-e_{j}\right)\right.
\end{aligned}
$$

then the corresponding expectation value of $\psi_{\mathrm{T}}$ with $\mathrm{H}^{*}$ is

$$
\left\langle\psi_{\mathrm{T}}\left|\mathrm{H}^{*}\right| \psi_{\mathrm{T}}\right\rangle=\left(1 / \Delta \mathrm{e}^{4}\left\{-\mathrm{e}_{i}\left\langle i\left|\left(\mathrm{H}-\mathrm{e}_{i}\right)^{2}\right| i\right\rangle^{2}-2\left\langle i\left|\left(\mathrm{H}-\mathrm{e}_{i}\right)^{2}\right| i\right\rangle\right.\right.
$$




$$
\begin{aligned}
& x<i\left|\left(H-e_{i}\right)^{3}\right| i>+e_{i}<i\left|\left(H-e_{i}\right)^{4}\right| i>+<i\left|\left(H-e_{i}\right)^{5}\right| i> \\
& -2 \sum_{n \neq i}^{M}\left\langle i\left|\left(H-e_{i}\right)^{3}\right| n>\left\langle n\left|\left(H-e_{i}\right)^{2}\right| i>\right.\right. \\
& \left.{ }_{n \neq i}^{M}|<i|\left(H-e_{i}\right)^{2}|n>|^{2}\left(e_{n}-2 e_{i}\right)\right\} \\
& +(1 / \Delta e)^{3}\left\{-4<i\left|\left(H-e_{i}\right)^{2}\right| i>+4 e_{i}<i\left|\left(H-e_{i}\right)^{3}\right| i>\right. \\
& +4<i\left|\left(H-e_{i}\right)^{4}\right| i>+\sum_{n \neq i}\left[-2|<i|\left(H-e_{i}\right)^{2}|n>|^{2}+2<i\left|\left(H-e_{i}\right)^{3}\right| n>\right. \\
& \left.\left.x<n\left|\left(H-e_{i}\right)^{2}\right| i>/\left(e_{i}-e_{n}\right)\right]\right\} \\
& +(1 / \Delta)^{2}\left\{4 e_{i}<i\left|\left(H-e_{i}\right)^{2}\right| i>+6<i\left|\left(H-e_{i}\right)^{3}\right| i>\right. \\
& \quad M \\
& +\sum_{n}\left[3 /\left(e_{i}-e_{n}\right)+e_{i} /\left(e_{i}-e_{n}\right)^{2}\right]|<i|\left(H-e_{i}\right)^{2}|n>|^{2}+e_{i} \\
& n \neq i-1 \\
& \left.-\sum_{j} e_{j}|<j|\left(H-e_{i}\right)^{2}|i>|^{2} /\left(e_{i}-e_{j}\right)^{2}\right\}+4<i\left|\left(H-e_{i}\right)^{2}\right| i>/ \Delta e \\
& =0
\end{aligned}
$$

Again details of the intermediate steps used to obtain (2.3.6) can be found in Appendix 1B. Substituting (2.3.3) and (2.3.6) back into (2.3.1) finally yields an expression for $W$ in terms of variable parameter $\Delta e$.

The solution of $d W / d(\Delta e)=0$ will then give an approximate optimal constant denominator, $\Delta e$. This solution is obtained directly by the computational symbolic program, Maple ${ }^{[50]}$. The details of the deduction of $W$ and $d W / d(\Delta e)$ by Maple can be found in Appendix 3. 


\subsection{Anharmonic Oscillator}

For the harmonic oscillator the schrödinger equation can be solved analytically, the resulting first five eigenfunctions are then

$$
\begin{aligned}
& \phi_{0}=N_{0} \exp \left(-x^{2} / 2\right) \\
& \phi_{1}=N_{1} \operatorname{xexp}\left(-x^{2} / 2\right) \\
& \phi_{2}=N_{2}\left(-1+2 x^{2}\right) \exp \left(-x^{2} / 2\right) \\
& \phi_{3}=N_{3}\left(-3 x+2 x^{3}\right) \exp \left(-x^{2} / 2\right) \\
& \phi_{4}=N_{4}\left(3-12 x^{2}+4 x^{4}\right) \exp \left(-x^{2} / 2\right)
\end{aligned}
$$

where the $\mathrm{N}_{i}(i=0,1,2,3,4)$ are normalization factors. For small $l$ and $m$, the functions above can be taken as approximate eigenfunctions of the anharmonic oscillator

$$
H=(1 / 2) p^{2}+(1 / 2) x^{2}+1 x^{3}+m x^{4}
$$

If the exponential coefficients can be variationally changed and the functions are kept orthonormal, an improved set of approximate eigenfunctions is obtained with

$$
\begin{aligned}
& \phi_{0}=N_{0} \exp \left(-r_{0} x^{2} / 2\right) \\
& \phi_{1}=N_{1} x \exp \left(-r_{1} x^{2} / 2\right) \\
& \phi_{2}=N_{2}\left[1-\left(r_{0}+r_{2}\right) x^{2}\right] \exp \left(-r_{2} x^{2} / 2\right) \\
& \phi_{3}=N_{3}\left[x-\left(r_{1}+r_{3}\right) x^{3} / 3\right] \exp \left(-r_{3} x^{2} / 2\right) \\
& \phi_{4}=N_{4}\left[1+b_{2} x^{2}+b_{4} x^{4}\right] \exp \left(-r_{4} x^{2} / 2\right)
\end{aligned}
$$


where $b_{2}$ and $b_{4}$ determined by orthogonality conditions are given by

$$
\begin{aligned}
b_{2}= & -\left(6 r_{4} r_{2} r_{0}+6 r_{4}^{2} r_{2}+r_{4} r_{2}^{2}+2 r_{4}^{2} r_{0}-r_{2}^{2} r_{0}+9 r_{0}^{2} r_{4}+4 r_{0}^{2} r_{2}+5 r_{0}^{3}\right) / \\
& \left(r_{4} r_{0}+r_{0} r_{2}-2 r_{2}^{2}+5 r_{0}^{2}+3 r_{4} r_{2}\right) \\
b_{4}= & -\left(r_{0}+r_{4}\right)^{2} / 3+(1 / 3)\left(r_{0}+r_{4}\right)\left(6 r_{4} r_{2} r_{0}+6 r_{4}^{2} r_{0}+r_{4} r_{2}^{2}+2 r_{4}^{2} r_{0}\right. \\
& \left.-r_{2}^{2} r_{0}+9 r_{0}^{2} r_{4}+4 r_{0}^{2} r_{2}+5 r_{0}^{3}\right) /\left(r_{4} r_{0}+r_{0} r_{2}-2 r_{2}^{2}+5 r_{0}^{2}+3 r_{4} r_{2}\right)
\end{aligned}
$$

The nonlinear optimization of the exponents $\left\{r_{i} ; i=0,\right.$. $\ldots, 4\}$ is carried out using Maple and the variational principle (2.3.1).

$$
\begin{aligned}
W_{i} & =\left\langle\phi_{i}\left|\left\langle H-\sum_{j=0}^{i-1} \varepsilon_{j} \mid \phi_{j}\right\rangle\left\langle\phi_{j}\right|\right) \mid \phi_{i}\right\rangle \mid\left\langle\phi_{i} \mid \phi_{i}\right\rangle \\
& =\left\langle\phi_{i}|H| \phi_{i}>-\sum_{j=0}^{i-1} \varepsilon_{j}\left\langle\phi_{i} \mid \phi_{j}\right\rangle\left\langle\phi_{j} \mid \phi_{i}\right\rangle\right. \\
& =\left\langle\phi_{i}|H| \phi_{i}\right\rangle
\end{aligned}
$$

where the orthonormal property $\left\langle\phi_{i} \mid \phi_{j}\right\rangle=\delta_{i j}$ has been used. Details of this symbolic computation are given in Appendix 3G. Once this optimized set of basis functions has been determined the corresponding zero order reference functions which form the model space can be obtained by linear variation.

$$
\begin{aligned}
& e_{i}=\langle i|H| i\rangle /\langle i \mid i\rangle \\
& |i\rangle=\sum_{j=0}^{4} b_{i j}\left|\phi_{j}\right\rangle \quad(i=0, \ldots, 4)
\end{aligned}
$$


Matrix elements required in equation (2.4.14) can be obtained by the integral program intt1 in Appendix 3C. The diagonalization program used to obtain corresponding eigenvalues and eigenvectors is given by the Fortran program eisg in Appendix 2 . 


\subsection{Introduction}

In Chapter 2 a multi-reference constant denominator perturbation theory (CDPT) was developed. The general purpose of this theory is to reduce incomplete basis set errors arising when solving the schrödinger equation with a finite basis set. As a first step, the theory derived, has been restricted to one particle Hamiltonians. Since this is a perturbation method, a major interest is then in its convergence. In order to examine this question, the anharmonic oscillator

$$
H=p^{2} / 2+x^{2} / 2+1 x^{3}+m x^{4}
$$

has been studied in five different cases using the reference functions given in section 2.4. First, the effects of the size and type of perturbation used are examined both for the variational choice of $\Delta e$ and the Padé approximant method. Secondly, the effects of the size of the multi-reference model space employed in this method are examined for the ground state. Finally, conclusions as well as suggestions of how this multi-reference CDPT approach can be extended to many particle systems are discussed.

\subsection{Effects of The Size and Type of Perturbation on The Convergence of CDPT}

A: Variational Results

Energy corrections calculated to fifth order are presented 
in Tables $3.1 \mathrm{a}$ and $3.1 \mathrm{~b}$ for the symmetric potentials $1=0$, $\mathrm{m}=0.1 ; 1=0, \mathrm{~m}=10$ and asymmetric potentials $1=0.1, \mathrm{~m}=0.1$; l=1.0, $\mathrm{m}=1.0$ respectively. Plots of these potentials along with the harmonic case $I=0, m=0$ are shown in Eig. 3.1 . The average energy $\Delta e$ used in these calculation is from variational method with trial function $\psi_{\mathrm{T}}=\psi_{0}+\psi_{1}+\psi_{2}$.

From the resulting perturbation series it can be seen that the first five energy corrections converge rapidly, especially the fifth energy correction, $E_{5}$. Comparing the sum of the first five energy corrections with variational results, $W$, we can see that such a energy correction series converges to a value slightly better than the variational result while still an upper bound to the exact energy.

Although in the model space five functions (see eqn. $(2.4 .6)-(2.4 .15)$ in section 2.4) have been used, in the quartic oscillator case (i.e. $l=0)$, only 3 functions make contributions to the ground and second excited state energies while 2 functions figure in the first excited state due to symmetric restrictions. For $m=0.1$ it is seen that the convergence is much better than the $m=10$ case no matter whether ground or excited is being calculated. For example, the ground state energy for $m=0.1$, improves after perturbation by more than 2 orders of magnitude. However the corresponding result for $m=10$ shows a markedly smaller improvement of about a factor twenty. This result is due to two related factors. The first is that the starting point $E_{0}+E_{1}$ determined by the reference state in the model space is 
Table 3.1a The Energy Corrections of CDPT for the Anharmonic Oscillator having a symmetric Potential

\begin{tabular}{|c|c|c|c|}
\hline & $\mathrm{n}=0$ & $n=1$ & $\mathrm{n}=2$ \\
\hline$E_{0}+E_{1}$ & 0.55922078 & 1.7732529 & 3.1468516 \\
\hline & $-0.60568377 \times 10^{-4}$ & $-0.28331632 \times 10^{-3}$ & $-0.62 \overline{2317408 \times 10^{-2}}$ \\
\hline $\mathrm{E}_{3}$ & $-0.10638020 \times 10^{-4}$ & $-0.61352147 \times 10^{-3}$ & $-0.13396620 \times 10^{-2}$ \\
\hline $\mathrm{E}_{4}$ & $-0.27372457 \times 10^{-5}$ & $-0.25310562 \times 10^{-3}$ & $-0.55052507 \times 10^{-3}$ \\
\hline$E_{5}$ & $-0.42001722 \times 10^{-8}$ & $0.12257083 \times 10^{-6}$ & $-0.55738406 \times 10^{-6}$ \\
\hline Sum & 0.55914681 & $1.7695 \underline{532}$ & $3.138 \underline{7302}$ \\
\hline W & 0.55914682 & $1.7695 \underline{537}$ & 3.1387322 \\
\hline Exact & 0.55914633 & 1.7695026 & 3.1386240 \\
\hline \multicolumn{4}{|c|}{$I=0, m=10$} \\
\hline & $\mathrm{n}=0$ & $\mathrm{n}=1$ & $\mathrm{n}=2$ \\
\hline$E_{0}+E_{1}$ & $1.50 \underline{93503}$ & 5.3763776 & $10 . \underline{445903}$ \\
\hline & $-0.28 \overline{90799 \times 10^{-2}}$ & $-0.3 \overline{581007} \times 10^{-1}$ & $-0.6 \overline{589216 \times 10^{-1}}$ \\
\hline$E_{3}$ & $-0.8190135 \times 10^{-3}$ & $-0.9909824 \times 10^{-2}$ & $-0.1762156 \times 10^{-1}$ \\
\hline $\mathrm{E}_{4}$ & $-0.4629495 \times 10^{-3}$ & $-0.6667632 \times 10^{-2}$ & $-0.1111389 \times 10^{-1}$ \\
\hline $\mathrm{E}_{5}$ & $-0.7185399 \times 10^{-6}$ & $0.8485106 \times 10^{-7}$ & $0.1498332 \times 10^{-4}$ \\
\hline Sum & $1.50 \underline{51768}$ & $5.32 \underline{46192}$ & 10.3512904 \\
\hline$W$ & $1.50 \underline{51776}$ & $5.32 \underline{46453}$ & $10 . \underline{\underline{513576}}$ \\
\hline Exact & 1.504972 & 5.321608 & 10.34706 \\
\hline
\end{tabular}

Note: 1.The average energy $\Delta e$ used in the calculation is from Rayleigh Ritz variational principle with trial function $\psi_{\mathrm{T}}=\psi_{0}+\psi_{1}+\psi_{2}$

2. Sum $=E_{0}+E_{1}+E_{2}+E_{3}+E_{4}+E_{5}$

3. W is the variational energy. 4. The exact results are from ref.39. 
Table 3.1b The Energy Corrections of CDPT for the Anharmonic oscillator having an Asymmetric Potential

\begin{tabular}{|c|c|c|c|}
\hline & $\mathrm{n}=0$ & $\mathrm{n}=1$ & $n=2$ \\
\hline$E_{0}+E_{1}$ & 0.55360114 & 1.7475173 & 3.1086418 \\
\hline $\mathrm{E}_{2}$ & $-0.66552910 \times 10^{-4}$ & $-0.14 \overline{701598} \times 10^{-2}$ & $-0 . \overline{94541810} \times 10^{-2}$ \\
\hline$E_{3}$ & $-0.13223122 \times 10^{-4}$ & $-0.30743279 \times 10^{-3}$ & $-0.21596789 \times 10^{-2}$ \\
\hline$E_{4}$ & $-0.39711597 \times 10^{-5}$ & $-0.15770162 \times 10^{-3}$ & $-0.11273851 \times 10^{-2}$ \\
\hline$E_{5}$ & $-0.24159471 \times 10^{-6}$ & $-0.12585386 \times 10^{-5}$ & $-0.43687582 \times 10^{-5}$ \\
\hline Sum & $0.55351 \underline{714}$ & $1.7455 \underline{8075}$ & 3.09589619 \\
\hline$W$ & $0.55351 \underline{716}$ & 1.74558207 & $3.095 \underline{89619}$ \\
\hline Exact & 0.55351618 & 1.7455093 & 3.0953972 \\
\hline \multicolumn{4}{|c|}{$I=1.0, m=1.0$} \\
\hline & $\mathrm{n}=0$ & $\mathrm{n}=1$ & $\mathrm{n}=2$ \\
\hline$E_{0}+E_{1}$ & $0.72 \underline{135734}$ & $2.50 \underline{32680}$ & $4 . \underline{96893420}$ \\
\hline$E_{2}$ & $-0.553754 \times 10^{-3}$ & $-0.13125 \times 10^{-2}$ & $-0 . \overline{06860951}$ \\
\hline $\mathrm{E}_{3}$ & $-0.178945 \times 10^{-3}$ & $-0.36827 \times 10^{-3}$ & -0.02294799 \\
\hline$E_{4}$ & $-0.104622 \times 10^{-3}$ & $-0.15981 \times 10^{-3}$ & -0.01667071 \\
\hline$E_{5}$ & $-0.1593 \times 10^{-6}$ & $-0.28195 \times 10^{-6}$ & $-0.41506 \times 10^{-3}$ \\
\hline Sum & $0.720 \underline{51986}$ & $2.501 \underline{4271}$ & $4.8 \underline{602909}$ \\
\hline W & $0.720 \underline{52003}$ & $2.501 \underline{4275}$ & $4.8 \underline{608645}$ \\
\hline Exact & 0.72046305 & 2.5013595 & 4.8386778 \\
\hline
\end{tabular}

Note: 1.The average energy $\Delta e$ used in the calculation is from Rayleigh Ritz variational principle with trial function $\psi_{\mathrm{T}}=\psi_{0}+\psi_{1}+\psi_{2}$

2. Sum $=E_{0}+E_{1}+E_{2}+E_{3}+E_{4}+E_{5}$

3.W is the variational energy.

4. The exact results are from ref. 39 . 

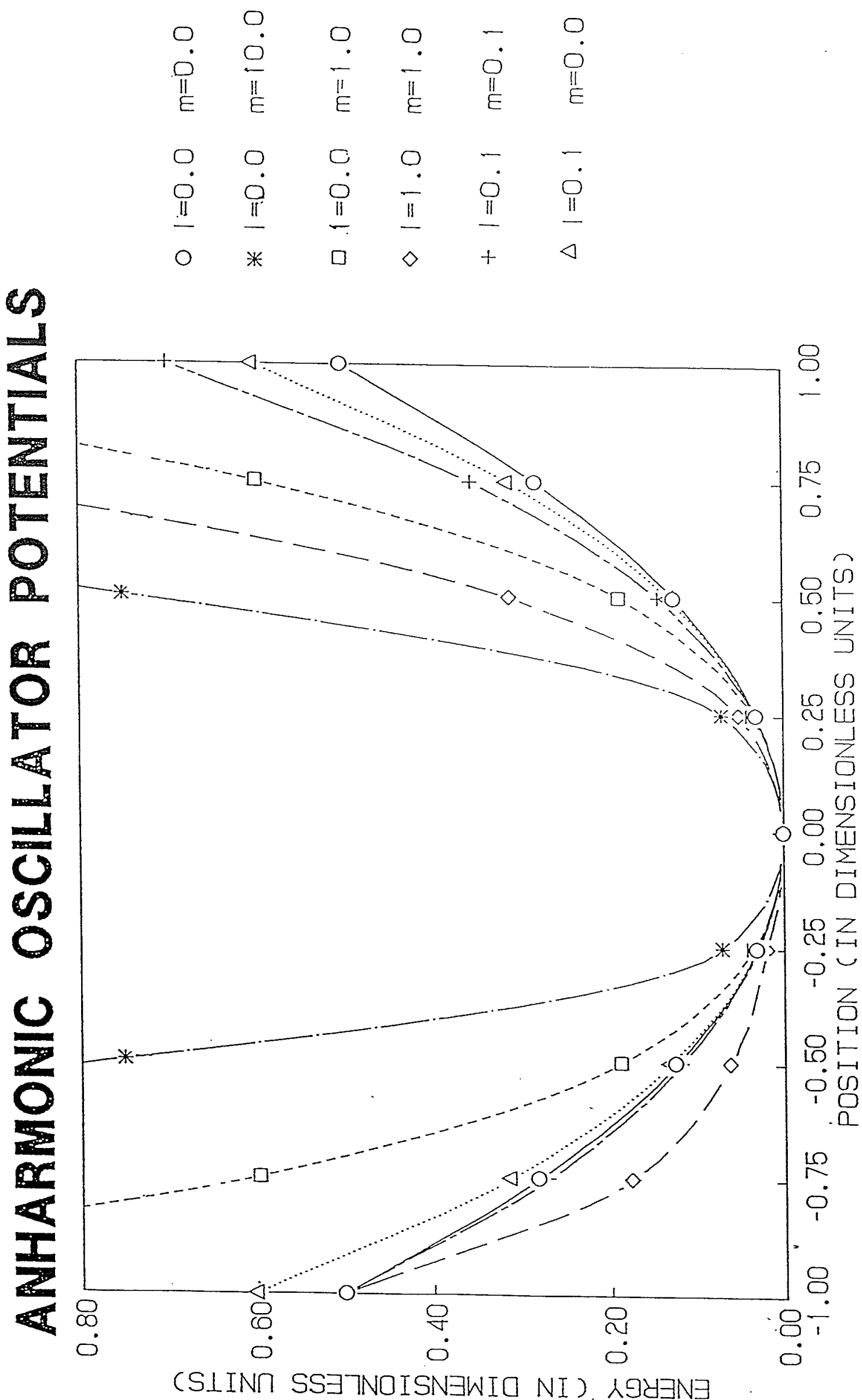
worse for $m=10$. The second is that the perturbation for $m$ $=10$ is much larger. Since the functional form of the model space basis set is derived from the eigenfunctions of the harmonic oscillator, one can take the difference between eigenvalues of harmonic and anharmonic oscillators as a rough measure of the size of the perturbation. For the harmonic oscillator, the first three energy eigenvalues are $0.5,1.5$ and 2.5. In comparison for $\mathrm{m}=0.1$ the exact energies are higher by $0.05914633,0.2695026$ and 0.638624 , Similarly for $m=10$, the differences are $1.004972,3.821608$ and 7.84706 . One might therefore expect slower convergence for the larger anharmonicity. This also explains why the ground state has a faster convergence than the excited states.

In Table $3.1 \mathrm{~b}$ the change of convergence with $I$ and $\mathrm{m}$ is $\mathrm{a}$ slightly different from that in Table 3.1a. The excited states, especially, have much worse convergence than the ground state. For example, the second excited state, in the case of $1=0.1 ; m=0.1$, has an improved energy by more than two orders of magnitude. However, for the $1=1.0 ; \mathrm{m}=1.0$ case, only an improvement of less than one order of magnitude has been achieved after the perturbation treatment.

The effects of $I$ and $m$ on convergence are more serious than for the symmetric potential. Consider the two cases $I=0.1$, $m=0.1$ in Table $3.1 \mathrm{~b}$ and $1=0, m=0.1$ in Table 3.1a. Although the asymmetric case has an energy closer to the harmonic oscillator than the symmetric case, the perturbation series for the symmetric case has the faster convergence rate. For instance, in the case of the ground states of the two cases, 
the energy corrections for the symmetric case give one more order of improvement than the asymmetric case. The explanation for this could be that the more complicated potential (see Fig. 3.1) should have a more complicated eigenfunction which is more difficult to approach in a perturbation treatment with the model space used.

\section{$B: \underline{\text { Pade Approximant Results }}$}

In Table 3.2 results with different constant denominators $\Delta e$, are shown to see how $\Delta e$ affects the rate of convergence. One quantity, $\rho$, used to measure the relative remaining incomplete basis set error is defined as $\rho=$ (final result-Exact) $\times 100 /$ (Starting value-Exact) (3.1.1)

Consider the second excited state of $I=0.1$ and $m=0.1$ in Table 3.2 as an example. The resulting energy perturbation expansions are very much dependent on the average energy $\Delta e$ used. The first series with $\Delta e=-6.00$ is fluctuating and its perturbation energy at fifth order has a $\rho$ of $14.15 \%$. In other words the incomplete basis set error has been reduced by $100 \%-14.15 \%=85.85 \%$. The second series with $\Delta e=$ -8.465 which is from the variational method gives the best result. The third series with $\Delta e=-10$ is a smooth monotonically decreasing series which has removed the incomplete basis set error at fifth order by $95.02 \%$. Although these three different expansions give three different results, the application of Pade approximants to these series 
Table 3.2. The Perturbation Energy Corrections with Different Average Energy $\Delta e$ and the Results after Pade

The ground state of $1=0.1, \mathrm{~m}=0.1$

\begin{tabular}{|c|c|c|c|}
\hline & $\mathrm{a}$ & $\mathrm{b}$ & $c$ \\
\hline$\Delta \mathrm{e}$ & -9.000 & $-11.281^{\star}$ & -13.000 \\
\hline$E_{0}+E_{1}$ & 0.55360114 & 0.55360114 & 0.55360114 \\
\hline $\mathrm{E}_{2}$ & $-0.83420759 \times 10^{-4}$ & $-0.665 \overline{52909 \times 10^{-4}}$ & $-0.577 \overline{52363 \times 10^{-4}}$ \\
\hline$E_{3}$ & $0.36778752 \times 10^{-6}$ & $-0.13223122 \times 10^{-4}$ & $-0.17594170 \times 10^{-4}$ \\
\hline $\mathrm{E}_{4}$ & $-0.26482576 \times 10^{-5}$ & $-0.39711597 \times 10^{-5}$ & $-0.18271209 \times 10^{-5}$ \\
\hline$E_{5}$ & $0.27326362 \times 10^{-5}$ & $-0.24159472 \times 10^{-6}$ & $-0.18271209 \times 10^{-5}$ \\
\hline Sum & 0.55351817 & 0.55351715 & 0.55351773 \\
\hline$\rho 1$ & $2.3440 \%$ & $1.1431 \%$ & $1.8225 \%$ \\
\hline$E[1,0]$ & 0.55351801 & 0.55351809 & 0.55351809 \\
\hline$E[2,1]$ & 0.55351677 & 0.55351676 & 0.55351676 \\
\hline$W[1,0]$ & 0.55351675 & 0.55351674 & 0.55351673 \\
\hline Exact & 0.55351618 & 0.55351618 & 0.55351618 \\
\hline$\rho 2$ & $0.6677 \%$ & $0.6590 \%$ & $0.6524 \%$ \\
\hline
\end{tabular}

The first excited state of $1=0.1, \mathrm{~m}=0.1$

\begin{tabular}{|c|c|c|c|}
\hline & a & $\mathrm{b}$ & $\mathrm{C}$ \\
\hline$\Delta \mathrm{e}$ & -6.000 & $-8.850^{\star}$ & -10.000 \\
\hline$E_{0}+E_{1}$ & 1.7475173 & 1.7475173 & 1.7475173 \\
\hline $\mathrm{E}_{2}$ & $-0.21684749 \times 10^{-2}$ & $-0.14701598 \times 10^{-2}$ & $-0.13010940 \times 10^{-2}$ \\
\hline$E_{3}$ & $0.36115630 \times 10^{-3}$ & $-0.30743279 \times 10^{-3}$ & $-0.39041225 \times 10^{-3}$ \\
\hline $\mathrm{E}_{4}$ & $-0.35991203 \times 10^{-3}$ & $-0.15770162 \times 10^{-3}$ & $-0.18189980 \times 10^{-3}$ \\
\hline$E_{5}$ & $0.50444060 \times 10^{-3}$ & $-0.12585386 \times 10^{-5}$ & $-0.50517718 \times 10^{-4}$ \\
\hline Sum & 1.74585451 & $1.7455 \underline{8075}$ & $1.7455 \underline{9338}$ \\
\hline$\rho 1$ & $17.1918 \%$ & $3.5581 \%$ & $4.1871 \%$ \\
\hline$E[1,0]$ & $1.745 \underline{65842}$ & $1.745 \underline{65842}$ & $1.745 \underline{65842}$ \\
\hline$E[2,1]$ & 1.74555819 & $1.7455 \underline{5671}$ & $1.7455 \underline{5610}$ \\
\hline$W[1,0]$ & $1.7455 \underline{5248}$ & $1.7455 \underline{5083}$ & $1.7455 \underline{5014}$ \\
\hline Exact & 1.7455093 & 1.7455093 & 1.7455093 \\
\hline$\rho 2$ & $2.1504 \frac{\circ}{6}$ & $2.0680 \%$ & $2.0340 \%$ \\
\hline
\end{tabular}


Table 3.2 (continued) The Perturbation energy Corrections with Different Average $\Delta e$ and the Results after Pade

The second excited state of $1=0.1, \mathrm{~m}=0.1$

\begin{tabular}{|c|c|c|c|}
\hline & a & $\mathrm{b}$ & $c$ \\
\hline$\Delta e$ & -6.000 & $-8.465^{*}$ & -10.000 \\
\hline$E_{0}+E_{1}$ & 3.1086418 & 3.1086418 & 3.1086418 \\
\hline$E_{2}$ & $-0.13338267 \times 10^{-1}$ & $-0 . \overline{94541810} \times 10^{-2}$ & $-0.80029664 \times 10^{-2}$ \\
\hline$E_{3}$ & $0.11810638 \times 10^{-2}$ & $-0.21596789 \times 10^{-2}$ & $-0.27759973 \times 10^{-2}$ \\
\hline$E_{4}$ & $-0.18851850 \times 10^{-2}$ & $-0.11273851 \times 10^{-2}$ & $-0.13474865 \times 10^{-2}$ \\
\hline $\mathrm{E}_{5}$ & $0.26716645 \times 10^{-2}$ & $-0.43687582 \times 10^{-5}$ & $-0.45860194 \times 10^{-3}$ \\
\hline Sum & 3.09727108 & 3.09589618 & 3.09605675 \\
\hline$\rho 1$ & $14.1482 \%$ & $3.7675 \%$ & $4.9798 \%$ \\
\hline$E[1,0]$ & $3.09 \underline{638852}$ & 3.09638853 & 3.09638853 \\
\hline$E[2,1]$ & 3.09570475 & 3.09569730 & 3.09569300 \\
\hline$W[1,0]$ & 3.09566358 & 3.09565597 & $3.095 \underline{65115}$ \\
\hline Exact & 3.0953972 & 3.0953972 & 3.0953972 \\
\hline$\rho 2$ & $2.0112 \%$ & 1.9538 웅 & $1.9173 \%$ \\
\hline
\end{tabular}

Note: 1.The average energy $\Delta e$ with star is from variational method with trial function $\psi_{\mathrm{T}}=\psi_{0}+\psi_{1}+\psi_{2} \cdot$

2. Sum $=E_{0}+E_{1}+E_{2}+E_{3}+E_{4}+E_{5}$.

$3 . \rho 1=($ Exact $-\operatorname{Sum}) \times 100 /\left(\operatorname{Exact}-\left(E_{0}+E_{1}\right)\right)$.

$4 \cdot p^{2}=(\operatorname{Exact}-W[1,0]) \times 100 /\left(\operatorname{Exact}-\left(E_{0}+E_{1}\right)\right)$.

5. Exact results are from ref. 39 .

yields results with almost the same energy. For the case of E[1,0] which involves energy corrections to third order, from the equations (2.2.17) and (2.2.18) in Chapter 2, it can be seen that this Pade approximant is strictly invariant to any denominator shifts. This can be seen in Table 3.2. The 
fourth and fifth order energy corrections, $E_{4}$ and $E_{5}$ r however, are dependent on the all reference functions in the model space. Hence the $E[2,1]$ Pade which uses these corrections shows a slight variance with $\Delta e$, especially for excited states. The double Pade, $w[1,0]$, defined by eq. $(2.1 .25)-(2.1 .32)$ is found to be the most successful method for removing the incomplete basis set error. It also shows very little dependence on the $\Delta e$ value. Take the second excited state of $1=0.1$ and $\mathrm{m}=0.1$ as an example again. The incomplete basis set error has been reduced by about 98\% after the application of the double Pade. The double Pade not only improved the results for the "a" and " $c$ " series but also for the "b" series which uses $\Delta e$ from the variational method. This fact shows that the double Pade is a good technique to process the perturbation series to obtain an accurate estimate of the energy.

C: General Results

Table $3.3 a$ presents the energies of the first three states for five different potentials which include the symmetric cases $(I=0, m=0.1,1.0,10)$ and asymmetric cases $(I=0.1$, $\mathrm{m}=0.1 ; 1=1.0, \mathrm{~m}=1.0)$ in $(1.4 .1)$.

For symmetric cases if $\mathrm{m}$ increases the relative remaining incomplete basis set error $\rho$ increases. For excited states almost the same values of $p$ are observed. In general for the symmetric potentials in Table $3.3 a$ the relative incomplete 


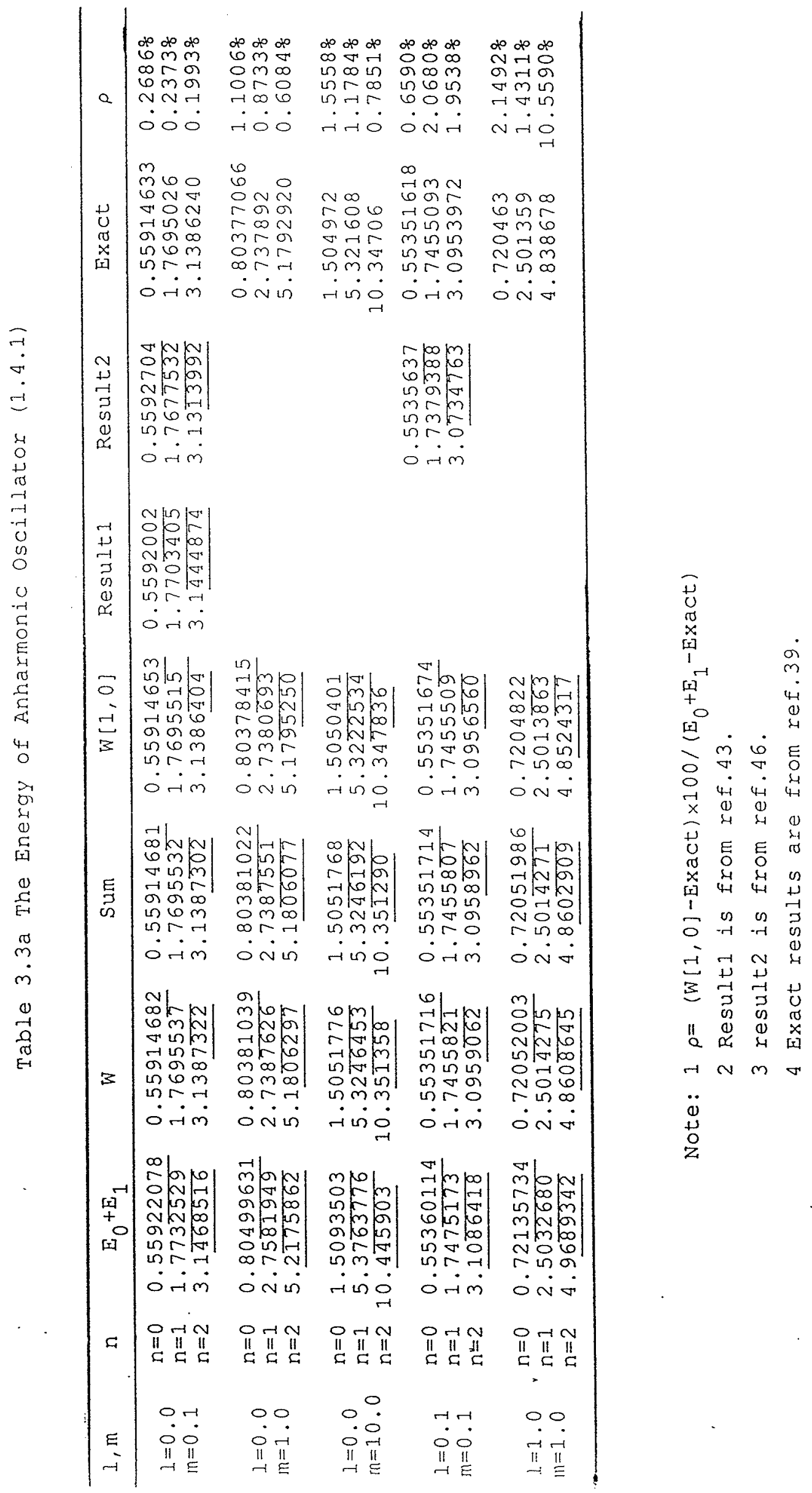


basis set errors of the ground state and the the excited states have been reduced by $98-99 \%$.

For the asymmetric potential, results are inferior to those found in the symmetric cases. Again the excited state energies are not as accurate as the ground state. The worst case is the second excited state of $1=1.0, \mathrm{~m}=1.0$, which has a 0.130256 energy difference between starting and exact points. Its relative incomplete basis set error, however, still has been reduced by $89.45 \%$

The results of Patnaik $[43]$ and Lai's ${ }^{[48]}$ are also presented in Table 3.3 a for comparison.

In Patnaik's work, a two step method is used, in which 19 basis functions are optimally selected and then a perturbation treatment is carried out to third order. Below we compare his results with ours at third order for the case l=0, $m=0.1$ with variational optimized $\Delta$ e (see Table $3.3 b$ ).

Table 3.3b The Comparison of Perturbation Energies at The Third order

\begin{tabular}{|c|c|c|c|}
\hline & $n=0$ & $n=1$ & $n=2$ \\
\hline a & 0.55914729 & 1.769964185 & 3.13891332 \\
\hline $\mathrm{b}$ & $0.559 \underline{2002}$ & $1.7 \underline{703405}$ & 3.1444874 \\
\hline Exact & 0.55914633 & 1.7695026 & 3.1313992 \\
\hline
\end{tabular}

Note: 1.Results of type "a" are from the developed CDPT in Chapter 2 and the denominator $\Delta \mathrm{e}$ is from variational method with trial function $\psi_{\mathrm{T}}=\psi_{0}+\psi_{1}$.

2. Results of type "b" are from Patnaik's work. 
For the developed CDPT perturbation up to third order in Chapter 2 only one reference function is involved for each state in the calculation of the energy corrections. Although this theory is much simpler than Patnaik's work, comparison of results shows the developed CDPT method has much more accuracy.

The other results shown in Table $3.3 \mathrm{a}$ are $[8,8]$ Pade approximants from Lai's work ${ }^{[48]}$. As reviewed in section 1.4 such a calculation based on the hypervirial theorem involves the energy expansion in terms of the quartic coefficient $m$ to the $32^{\prime}$ th order. However these results are still unreliable.

D: The Effect of Perturbation Order

If only one wave function correction is used in the calculation, the energy perturbation can be carried out to third order with trial function $\psi_{\mathrm{T}}=\psi_{0}+\psi_{1}$. (see Appendix 3F) Table 3.4 presents such results for the $1=1.0, \mathrm{~m}=1.0$ asymmetric potential and compares them with those found from the two wave function corrections $\psi_{\mathrm{T}}=\psi_{0}+\psi_{1}+\psi_{2}$. In both types the average denominator, $\Delta e$ has been variationally optimized. In addition the same model space is used hence both have the same starting point $E_{0}+E_{1}$. For the perturbation treatments to third order the second order energy corrections, $E_{2}$, are larger in magnitude than those found in perturbation treatments carried out to fifth order because of the different variational $\Delta e$ used. Also the 
Table 3.4. The Comparison of Perturbation Energies between Third Order and Eifth Order in the Case $1=1.0, m=1.0$

\begin{tabular}{|c|c|c|c|c|}
\hline & 3rd Order & 5 th order & $3 r d$ Order & 5th Order \\
\hline$E_{0}+E_{1}$ & $0.72 \underline{135734}$ & $0.72 \underline{135734}$ & $2.50 \underline{32680}$ & 2.5032680 \\
\hline & $-0.818094 \times 10^{-2}$ & $-0.55{\overline{3754 \times 10^{-3}}}^{-3}$ & $-0.18{\overline{248 \times 10^{-2}}}^{-2}$ & $-0.13{\overline{125 \times 10^{-2}}}^{-2}$ \\
\hline$E_{3}$ & $-0.36 \times 10^{-7}$ & $-0.178945 \times 10^{-3}$ & $-0.41 \times 10^{-6}$ & $-0.36827 \times 10^{-3}$ \\
\hline$E_{4}$ & & $-0.104622 \times 10^{-3}$ & & $-0.15981 \times 10^{-3}$ \\
\hline$E_{5}$ & & $-0.1593 \times 10^{-6}$ & & $-0.28195 \times 10^{-6}$ \\
\hline Sum & $0.720 \underline{53925}$ & 0.72051986 & $2.501 \underline{4436}$ & 2.5014271 \\
\hline$W$ & $0.720 \underline{53921}$ & $0.720 \underline{52003}$ & $2.5014 \underline{4438}$ & $2.501 \underline{4275}$ \\
\hline$E[1,0]$ & $0.720 \underline{53921}$ & 0.72053921 & 2.50144436 & 2.5014436 \\
\hline$W[1,0]$ & & $0.7204 \underline{8222}$ & & $2.501 \overline{8863}$ \\
\hline Exact & 0.72046305 & 0.72046305 & 2.5013595 & 2.5013595 \\
\hline$\rho$ & $8.5162 \%$ & $2.1492 \%$ & $4.4053 \%$ & $1.4311 \%$ \\
\hline & 3rd Order & 5th Order & & \\
\hline$E_{0}+E_{1}$ & 4.96893420 & 4.96893420 & & \\
\hline $\mathrm{E}_{2}$ & -0.10222072 & -0.06860951 & & \\
\hline$E_{3}$ & $-0.86250 \times 10^{-3}$ & -0.02294799 & & \\
\hline $\mathrm{E}_{4}$ & & -0.01667071 & & \\
\hline$E_{5}$ & & $-0.41506 \times 10^{-3}$ & & \\
\hline Sum & $4.8 \underline{65851}$ & $4.8 \underline{602909}$ & & \\
\hline$W$ & 4.8667135 & $4.8 \underline{608645}$ & & \\
\hline$E[1,0]$ & $4.8 \underline{658436}$ & $4.8 \underline{658438}$ & & \\
\hline$W[1,0]$ & & $4.8 \underline{524317}$ & & \\
\hline Exact & 4.8386778 & 4.8386778 & & \\
\hline$\rho$ & $20.8557 \%$ & $10.5591 \%$ & & \\
\hline
\end{tabular}

Note.1. $\rho=(E[1,0]-E x a c t) \times 100 /\left(E_{0}+E_{1}-E x a c t\right)$ for the third order perturbation. $\rho=(W[1,0]-E x a c t) \times 100 /\left(E_{0}+E_{1}-E x a c t\right)$ for the fifth order perturbation 
resulting third order energy corrections, $E_{3}$, in the former case almost give no contribution to the energy. The $E[1,0]$ Pade approximants which are invariant to denominator shifts give identical energies for the two perturbation expansions.

Comparing the values of $\rho$, the relative remaining incomplete basis set exrors, one finds these have been reduced by a factor of between two and four as one goes from third to fifth order.

For excited states the accuracy of the third order series is much worse than the fifth order series compared with the ground state results. This is due to the slower convergence caused by a worse starting point and greater anharmonicity in the excited states.

\subsection{The Effect of Starting Point}

In the last several sections it has been observed that the starting point greatly influences the final result. In this section this point is examined for the ground state of anharmonic oscillator. In Table 3.5 results are presented for single reference and multi-reference model spaces. For the single reference model space (type "a" and "b") the general function

$$
f(x)=N\left(1+c_{1} x+c_{2} x^{2}\right) \exp \left(-r x^{2}\right)
$$


Table 3.5 The Effect of starting Point on the Ground states Energy

\begin{tabular}{|c|c|c|c|c|}
\hline & $E_{0}+E_{1}$ & $W[1,0]$ & Exact & $\rho$ \\
\hline \multirow[t]{2}{*}{$1=0.0$} & $0.5 \underline{60307}$ & $0.5591 \underline{57}$ & 0.55914633 & $0.9474 \%$ \\
\hline & $0.559 \underline{409}$ & $0.5591 \underline{57}$ & 0.55914633 & $4.1825 \%$ \\
\hline $\mathrm{m}=0.1 ; \mathrm{c}$ & $0.559 \underline{22078}$ & $0.559146 \underline{\underline{3}}$ & 0.55914633 & $0.2686 \%$ \\
\hline \multirow[t]{2}{*}{$I=0.0}$, & $0.8 \underline{12500}$ & $0.80 \underline{4105}$ & 0.80377066 & $3.8263 \%$ \\
\hline & $0.80 \underline{7415}$ & $0.80 \underline{4087}$ & 0.80377066 & $8.6718 \%$ \\
\hline $\mathrm{m}=1.0 ; \mathrm{c}$ & $0.80 \underline{499631}$ & $0.8037 \underline{8415}$ & 0.80377066 & $1.1006 \%$ \\
\hline \multirow{2}{*}{$\begin{aligned} & I=0.0, a \\
& b\end{aligned}$} & $1.5 \underline{31250}$ & $1.50 \underline{6410}$ & 1.504972 & $5.4723 \%$ \\
\hline & $1.5 \underline{17445}$ & $1.50 \underline{6237}$ & 1.504972 & $10.1420 \%$ \\
\hline$m=10 ; c$ & $1.50 \underline{93503}$ & $1.50 \underline{50401}$ & 1.504972 & $1.5558 \%$ \\
\hline \multirow{2}{*}{$\begin{aligned} & 1=0.1, a \\
& b\end{aligned}$} & $0.5 \underline{60307}$ & $0.55 \underline{4640}$ & 0.55351618 & $16.5513 \%$ \\
\hline & $0.553 \underline{953}$ & $0.5535 \underline{35}$ & 0.55351618 & $4.3478 \%$ \\
\hline $\mathrm{m}=0.1 ; \mathrm{c}$ & $0.553 \underline{60114}$ & $0.5535167 \underline{4}$ & 0.55351618 & $0.6590 \%$ \\
\hline \multirow{2}{*}{$\begin{array}{r}l=1.0, \\
b \\
b\end{array}$} & $0 . \underline{812500}$ & $0.7 \underline{50664}$ & 0.720463 & $32.8140 \%$ \\
\hline & $0.72 \underline{9672}$ & $0.72 \underline{1081}$ & 0.720463 & $6.7108 \%$ \\
\hline$m=1.0 ; c$ & $0.72 \underline{135734}$ & $0.7204 \underline{822}$ & 0.720463 & $2.1492 \%$ \\
\hline
\end{tabular}

Note: 1.W[1,0] is double Pade result.

2. $p=(W[1,0]-$ Exact $) \times 100 /\left(E_{0}+E_{1}-E x a c t\right)$.

3 . Exact results are from ref. 39 .

is used. For type "a" model space found in Table $3.5, c_{1}$ and $c_{2}$ are set to zero and the exponent is optimized. For type "b" model space, $c_{1}, c_{2}$ and $r$ are optimized. Type "c" uses the five reference functions from Chapter 2, section 2.4 .

Consider the first three symmetric potential cases. Although type "b" has a better starting point than type "a" the values of $w[1,0]$ for both types are almost same. Hence, in terms of the values of $\rho$ which gives a rough measure of the 
convergence of the perturbation expansion relative to the starting point, type "b" has the worst convergence. In comparison, the best starting reference function is the multi-reference one, type "c". This type always gives the best results in terms of relative convergence, $\rho$ and energy, $W[1,0]$

For the last two asymmetric potential cases, the effect of starting point is much more critical than in the symmetric cases. For example, the case $1=1.0, m=1.0$, the third type of reference functions gives improvement of accuracy by two decimal points with relative remaining error $\rho=2.1492 \%$. However the first type of reference function gives one decimal improvement to accuracy with relative remaining error $\rho=32.814 \%$. The possible reason is that more complicated potential will be more sensitive to the starting point.

\subsection{Conclusions}

In this thesis we have demonstrated that the developed CDPT can be successfully applied to the anharmonic oscillator (1.4.1). The advantage of this theory is that very few basis functions are needed and all calculations if carried out to high enough order in the perturbation treatment effectively use a complete basis set since the closure relation is used. If the trial function is $\psi_{\mathrm{T}}=\sum_{i=0}^{N} \psi_{i}$ in the developed CDPT and average energy $\Delta e$ is obtained by the variational method, the energy corrections calculated to the $2 \mathrm{~N}+1^{\prime}$ th order converge. The most accurate estimates are however the Pade approximant 
methods especially the double Pade. One interesting point of this theory is that in contrast to single reference perturbation theory multi-reference perturbation expansions have higher order $[n, n-1]$ Pade's $(n \geq 1)$ which are not invariant to scalings of the energy denominators. This results from the high order perturbation energy corrections being inhomogeneously dependent on $\Delta$ e (see Eq. (2.2.26) and $(2.2 .30))$

Considering the goal of the theory, inspection of Tables 3.2 through to 3.4 shows that the incomplete basis set error has been greatly reduced by the CDPT method. The choice of starting functions (or model space) is still very important since the only input into this theory is the basis set. The results of Table 3.5 have demonstrated this.

Further research of this multi-reference CDPT method could be done in two areas. One is the extension of the theory developed in Chapter 2 to the multi-particle case. A possible approach might be to get an effective potential for each particle and then reduce the Hamiltonian to a sum of effective one particle operators. After this step the CDPT could then be applied.

An example of this approach is the application of CDPT to the Hartree Fock method for atomic and molecular cases. Although the Hartree Fock operator is a one particle operator, there is a singularity problem in the calculation. This problem arises from the coulomb potential 1/r. In the calculation of energy by $\mathrm{CDPT}$ in chapter 2 the integral $\left\langle\mathrm{H}^{\mathrm{n}}\right\rangle$ $(\mathrm{n}=1,2,3, \ldots)$ cannot be avoided hence the integral $<1 / r^{n}>$ 
appears and gives the singularity problem. One way to remove this singularity is to use a variable denominator $\Delta e$ instead of a constant one. If so this theory could be extended and made more flexible. Such an approach limited to third order has been tried by Kirtmann ${ }^{[31]}$.

\section{Acknowledgement}

I would like to thank Dr. John Cullen for his help and encouragement in finishing this thesis. 
Appendix 1: The Deduction of the Eifth Order Perturbation

Energy Correction and Variational Energy

A: Deduction of the fifth order energy correction

In Chapter 2 the wave corrections $\left|\psi_{1}\right\rangle,\left|\psi_{2}\right\rangle$ and the $V\left|\psi_{1}>, V\right| \psi_{2}>$ are obtained as

$$
\begin{aligned}
\left|\psi_{1}\right\rangle= & (1 / \Delta e)\left(H-e_{i}\right) \mid i> \\
\left|\psi_{2}\right\rangle= & -(1 / \Delta e)^{2}\left(<i\left|\left(H-e_{i}\right)^{2}\right| i>\right)\left|i>+(1 / \Delta e)\left(H-e_{i}\right)\right| i> \\
& +(1 / \Delta e)^{2}\left(H-e_{i}\right)^{2}\left|i>+\sum_{n \neq i}^{M}\right| n>\left\langle n\left|\left(H-e_{i}\right)^{2}\right| i>\right. \\
& \times\left[1 /\left(e_{i}-e_{n}\right)-1 / \Delta e\right] / \Delta e
\end{aligned}
$$

$$
V \mid \psi_{1}>=\left[\left(H-e_{i}\right)+\left(H-e_{i}\right)^{2} / \Delta e \mid i>\right]
$$

$$
\begin{aligned}
V\left|\psi_{2}\right\rangle= & \sum_{n \neq i}^{M}\left(H-e_{n}\right) \mid n>\left\langle n\left|\left(H-e_{i}\right)^{2}\right| i>/\left[\left(e_{i}-e_{n}\right) \Delta e\right]\right. \\
& -(I / \Delta e)^{2} \sum_{n=0}^{M}\left(H-e_{n}\right) \mid n>\left\langle n\left|\left(H-e_{i}\right)^{2}\right| i\right\rangle
\end{aligned}
$$

$$
\begin{aligned}
& +1 / \Delta e)^{2}\left[\left(H-e_{i}\right)^{3}+2 \Delta e\left(H-e_{i}\right)^{2}+(\Delta e)^{2}\left(H-e_{i}\right)\right] \mid i> \\
& +(1 / \Delta e)^{2} \sum_{n=0}^{M}\left(e_{n}-e_{i}+\Delta e\right) \mid n>\left\langle n\left|\left(H-e_{i}\right)^{2}\right| i>\right.
\end{aligned}
$$

The norm of the first order wave function and its corresponding overlap with the second order wave function are then given by

$$
\left\langle\psi_{1} \mid \psi_{1}\right\rangle=(1 / \Delta e)^{2}<i\left|\left(H-e_{i}\right)^{2}\right| i>
$$




$$
\left\langle\psi_{1} \mid \psi_{2}\right\rangle=\left[\left\langle i\left|\left(H-e_{i}\right)^{2}\right| i>+<i\left|\left(H-e_{i}\right)^{3}\right| i>/ \Delta e\right] /(\Delta e)^{2}\right.
$$

Therefore the renormalization terms $E_{2}\left\langle\psi_{1} \mid \psi_{2}\right\rangle$ and $E_{3}\left\langle\psi_{1} \mid \psi_{1}\right\rangle$ found at fifth order in equation (2.1.21) become $E_{2}\left\langle\psi_{1}\right| \psi_{2}>=\left[\left\langle i\left|\left(H-e_{i}\right)^{2}\right| i\right\rangle^{2}+\left\langle i\left|\left(H-e_{i}\right)^{3}\right| i>\left\langle i\left|\left(H-e_{i}\right)^{2}\right| i>/ \Delta e\right] /(\Delta e)^{3}\right.\right.$ (A. 7)

$E_{3}<\psi_{1} \mid \psi_{1}>=\left[\left\langle i\left|\left(H-e_{i}\right)^{2}\right| i\right\rangle^{2}+\left\langle i\left|\left(H-e_{i}\right)^{3}\right| i>\left\langle i\left|\left(H-e_{i}\right)^{2}\right| i>/ \Delta e\right] /(\Delta e)^{3}\right.\right.$

The remaining fifth order term $\left\langle\psi_{2}\left|\left(V-E_{1}\right)\right| \psi_{2}\right\rangle$ is obtained by

$$
\begin{aligned}
& \left\langle\psi_{2}\left|V-E_{1}\right| \psi_{2}\right\rangle=\left\langle\psi_{2}|V| \psi_{2}\right\rangle \\
& =-(1 / \Delta e)^{4}<i\left|\left(H-e_{i}\right)^{2}\right| i>\left[<i\left|\left(H-e_{i}\right)^{3}\right| i>+2 \Delta e<i\left|\left(H-e_{i}\right)^{2}\right| i>\right] \\
& \left.+(1 / \Delta e)^{3}<i\left|\left(H-e_{i}\right)^{2}\right| i\right\rangle^{2}+(1 / \Delta e)^{2} \sum_{n \neq i}^{M}|<i|(H-e)^{2}|n>|^{2} \\
& \times\left[1 /\left(e_{i}-e_{n}\right)-1 / \Delta e\right] \\
& -(1 / \Delta e)^{3}\left\langle i\left|\left(H-e_{i}\right)^{2}\right| i\right\rangle^{2}+(1 / \Delta e)^{3}\left[\left\langle i\left|\left(H-e_{i}\right)^{4}\right| i\right\rangle\right. \\
& \left.+2 \Delta e<i\left|\left(H-e_{i}\right)^{3}\right| i>+(\Delta e)^{2}<i\left|\left(H-e_{i}\right)^{2}\right| i>\right] \\
& \left.+(1 / \Delta e)^{3} \sum_{n \neq i}^{M}\left[\left\langle i\left|\left(H-e_{i}\right)^{3}\right| n\right\rangle+\left(e_{i}-e_{n}\right)<i\left|\left(H-e_{i}\right)^{2}\right| n\right\rangle\right]\left\langle n\left|\left(H-e_{i}\right)^{2}\right| i\right\rangle \\
& \times\left[1 /\left(e_{i}-e_{n}\right)-1 / \Delta e\right]-(1 / \Delta e)^{4}<i\left|\left(H-e_{i}\right)^{3}\right| i><i\left|\left(H-e_{i}\right)^{2}\right| i> \\
& +(1 / \Delta e)^{4}\left[\left\langle i\left|\left(H-e_{i}\right)^{5}\right| i>+2 \Delta e<i\left|\left(H-e_{i}\right)^{4}\right| i>+(\Delta e)^{2}<i\left|\left(H-e_{i}\right)^{3}\right| i>\right]\right. \\
& -(1 / \Delta e) \sum_{n=0}^{4}\left(e_{n}-e_{i}+\Delta e\right)|<i|\left(H^{2}-e_{i}\right)^{2}|n>|^{2}
\end{aligned}
$$




$$
\begin{aligned}
& \left.+\sum_{m \neq i}^{M} \sum_{n \neq i}^{M}\left[1 /\left(e_{i}-e_{n}\right)-1 / \Delta e\right]<i\left|\left(H-e_{i}\right)^{2}\right| n\right\rangle\left\langle n\left|\left(H-e_{m}\right)\right| m\right\rangle\left\langle m\left|\left(H-e_{i}\right)^{2}\right| i\right\rangle \\
& x\left[1 /\left(e_{i}-e_{m}\right)-1 / \Delta e\right] / \Delta e \\
& +(1 / \Delta e)^{3} \sum_{n \neq i}^{M}\left[1 /\left(e_{i}-e_{n}\right)-1 / \Delta e\right]<i\left|\left(H-e_{i}\right)^{2}\right| n>\left[\left\langlen\left|\left(H-e_{i}\right)^{3}\right| i>\right.\right. \\
& \left.\left.+2 \Delta e<n\left|\left(H-e_{i}\right)^{2}\right| i>\right]-(1 / \Delta e)^{3} \sum_{n \neq i}^{M} \sum_{m=0}^{M}\left[1 /\left(e_{i}-e_{n}\right)-1 / \Delta e\right]<i\left|\left(H-e_{i}\right)^{2}\right| n\right\rangle \\
& x<n|m><m|\left(H-e_{i}\right)^{2} \mid i>\left(e_{m}-e_{i}+\Delta e\right) \\
& =-2 /(\Delta e)^{4}<i\left|\left(H-e_{i}\right)^{2}\right| i>\left\langle i\left|\left(H-e_{i}\right)^{3}\right| i>-3<i\left|\left(H-e_{i}\right)^{2}\right| i\right\rangle^{2} /(\Delta e)^{3} \\
& +(1 / \Delta e)^{2} \sum_{n \neq i}^{M}\left\{|<i|\left(H-e_{i}\right)^{2}|n>|^{2}\left[1 /\left(e_{i}-e_{n}\right)-1 / \Delta e\right]\left[2+2\left(e_{i}-e_{n}\right) / \Delta e\right]\right. \\
& +2\left[1 /\left(e_{i}-e_{n}\right)-1 / \Delta e\right]<i\left|\left(H-e_{i}\right)^{3}\right| n><n\left|\left(H-e_{i}\right)^{2}\right| i>/ \Delta e \\
& \left.-\left(e_{n}-e_{i}+\Delta e\right)|<i|\left(H-e_{i}\right)^{2}|n>|^{2} /(\Delta e)^{2}\right\}+3<i\left|\left(H-e_{i}\right)^{4}\right| i>/(\Delta e)^{3} \\
& +3<i\left|\left(H-e_{i}\right)^{3}\right| i>/(\Delta e)^{2}+\left\langle i\left|\left(H-e_{i}\right)^{2}\right| i>/ \Delta e+\left\langle i\left|\left(H-e_{i}\right)^{5}\right| i>/(\Delta e)^{4}\right.\right.
\end{aligned}
$$

Finally substituting equations (A.7), (A.8) and (A.9) results in

$$
\begin{aligned}
E_{5}= & \left\langle\psi_{2}\left|V-E_{1}\right| \psi_{2}>-E_{3}\left\langle\psi_{1}\right| \psi_{1}>-2 E_{2}\left\langle\psi_{1} \mid \psi_{2}\right\rangle\right. \\
= & -5<i\left|\left(H-e_{i}\right)^{2}\right| i>\left\langle i\left|\left(H-e_{i}\right)^{3}\right| i>/(\Delta e)^{4}-6<i\left|\left(H-e_{i}\right)^{2}\right| i\right\rangle^{2} /(\Delta e)^{3} \\
& +(1 / \Delta e)^{2} \sum_{n \neq i}^{M}\left\{|<i|\left(H-e_{i}\right)^{2}|n>|^{2}\left[1 /\left(e_{i}-e_{n}\right)-1 / \Delta e\right]\left[2+2\left(e_{i}-e_{n}\right) / \Delta e\right]\right. \\
& +2\left[1 /\left(e_{i}-e_{n}\right)-1 / \Delta e\right]<i\left|\left(H-e_{i}\right)^{3}\right| n>\left\langle n\left|\left(H-e_{i}\right)^{2}\right| i>/ \Delta e\right. \\
& \left.-\left(e_{n}-e_{i}+\Delta e\right)|<i|\left(H-e_{i}\right)^{2}|n>|^{2} /(\Delta e)^{2}\right\} \\
& +3<i\left|\left(H-e_{i}\right)^{4}\right| i>/(\Delta e)^{3}+3<i\left|\left(H-e_{i}\right)^{3}\right| i>/(\Delta e)^{2}+<i\left|\left(H-e_{i}\right)^{2}\right| i>/ \Delta e \\
& +<i\left|\left(H-e_{i}\right)^{5}\right| i>/(\Delta e)^{4}
\end{aligned}
$$


$\mathrm{B}$ : Deduction of $\mathrm{W}=\left\langle\psi_{\mathrm{T}}\left|\mathrm{H}^{\star}\right| \psi_{\mathrm{T}}\right\rangle /\left\langle\psi_{\mathrm{T}} \mid \psi_{\mathrm{T}}\right\rangle$

In section 2.3 the operator $\mathrm{H}^{*}$ and trial function $\psi_{\mathrm{T}}$ are defined as

$$
\begin{aligned}
H^{*} & =\underset{j=0}{\mathrm{H}-\sum_{j} e_{j}|j\rangle<j \mid} \\
\mid \psi_{T}> & =|i>+| \psi_{1}>+\mid \psi_{2}> \\
& =\left[1-<i\left|\left(H-e_{i}\right)^{2}\right| i>/(\Delta e)^{2}\right]\left|i>+2\left(H-e_{i}\right) / \Delta e\right| i> \\
& +\left(H-e_{i}\right)^{2} /(\Delta e)^{2}\left|i>+\sum_{n \neq i}^{M}\right| n>\left\langle n\left|\left(H-e_{i}\right)^{2}\right| i>\left[1 /\left(e_{i}-e_{n}\right)-1 / \Delta e\right] / \Delta e\right.
\end{aligned}
$$

Then

$$
\begin{aligned}
& \mathrm{H}^{*}\left|\psi_{\mathrm{T}}\right\rangle=e_{i}\left[1-(1 / \Delta e)^{2}\left\langle i\left|\left(H-e_{i}\right)^{2}\right| i\right\rangle\right]|i\rangle \\
& +\left[2 e_{i} / \Delta e+\left(1-<i\left|\left(H-e_{i}\right)^{2}\right| i>/(\Delta e)^{2}\right)\right]\left(H-e_{i}\right) \mid i> \\
& +\left[e_{i} /(\Delta e)^{2}+2 / \Delta e\right]\left(H-e_{i}\right)^{2}\left|i>+(1 / \Delta e)^{2}\left(H-e_{i}\right)^{3}\right| i> \\
& +\sum_{n \neq i}^{M}\left(H-e_{i}\right)|n><n|\left(H-e_{i}\right)^{2} \mid i>\left[1 /\left(e_{i}-e_{n}\right)-1 / \Delta e\right] / \Delta e \\
& +e_{i} \sum_{n \neq i}^{M}|n><n|\left(H-e_{i}\right)^{2} \mid i>\left[1 /\left(e_{i}-e_{n}\right)-1 / \Delta e\right] / \Delta e \\
& -(I / \Delta e) \sum_{j=0}^{i-1} e_{j}|j\rangle\left\langle j\left|\left(H-e_{i}\right)^{2}\right| i>/\left(e_{i}-e_{j}\right)\right.
\end{aligned}
$$

Substitution of $(B .1)$ and $(B .2)$ into $\left\langle\psi_{\mathrm{T}}\left|\mathrm{H}^{*}\right| \psi_{\mathrm{T}}\right\rangle$ results in

$$
\begin{aligned}
\left\langle\psi_{\mathrm{T}}\left|\mathrm{H}^{*}\right| \psi_{\mathrm{T}}\right\rangle= & e_{i}\left[1-(1 / \Delta \mathrm{e})^{2}<i\left|\left(H-e_{i}\right)^{2}\right| i>\right]^{2} \\
& +\left[1-(1 / \Delta \mathrm{e})^{2}<i\left|\left(H-e_{i}\right)^{2}\right| i>\right] \quad\left[e_{i} /(\Delta e)^{2}+2 / \Delta e\right] \\
& x<i\left|\left(H-e_{i}\right)^{2}\right| i>+\left[1-(1 / \Delta e)^{2}<i\left|\left(H-e_{i}\right)^{2}\right| i>\right] \\
& x<i\left|\left(H-e_{i}\right)^{3}\right| i>/(\Delta e)^{2}+(2 / \Delta e)
\end{aligned}
$$




$$
\begin{aligned}
& \times\left[\left(2 e_{i} / \Delta e\right)+\left(1-<i\left|\left(H-e_{i}\right)^{2}\right| i>/(\Delta e)^{2}\right)\right]\left\langle i\left|\left(H-e_{i}\right)^{2}\right| i\right\rangle \\
& +\left(2 / \Delta e^{2}\right)\left[e_{i} / \Delta e+2\right]<i\left|\left(H-e_{i}\right)^{3}\right| i>+2<i\left|\left(H-e_{i}\right)^{4}\right| i>/(\Delta e)^{3} \\
& +\left(2 / \Delta e^{2}\right) \sum_{n \neq i}^{M} \mid\left\langle i\left|\left(H-e_{i}\right)^{2}\right| n>\left.\right|^{2}\left[1 /\left(e_{i}-e_{n}\right)-1 / \Delta e\right]\right. \\
& +\left(e_{i} / \Delta e^{2}\right)\left[1-\left\langle i\left|\left(H-e_{i}\right)^{2}\right| i\right\rangle /(\Delta e)^{2}\right]\left\langle i\left|\left(H-e_{i}\right)^{2}\right| i\right\rangle \\
& +\left[2 e_{i} / \Delta e+\left(1-<i\left|\left(H-e_{i}\right)^{2}\right| i>/(\Delta e)^{2}\right)\right]<i\left|\left(H-e_{i}\right)^{3}\right| i>/(\Delta e)^{2} \\
& +\left[e_{i} / \Delta e+2\right]<i\left|\left(H-e_{i}\right)^{4}\right| i>/ \Delta e^{3}+<i\left|\left(H-e_{i}\right)^{5}\right| i>/ \Delta e^{4} \\
& +(1 / \Delta e)^{3} \sum_{n \neq i}^{M}<i\left|\left(H-e_{i}\right)^{3}\right| n><n\left|\left(H-e_{i}\right)^{2}\right| i>\left[1 /\left(e_{i}-e_{n}\right)-1 / \Delta e\right] \\
& +\left(e_{i} / \Delta e^{3}\right) \sum_{n \neq i}^{M}|<i|\left(H-e_{i}\right)^{2}|n>|^{2}\left[1 /\left(e_{i}-e_{n}\right)-1 / \Delta e\right] \\
& -(1 / \Delta e)^{3} \sum_{j=0}^{i-1} e_{j}|<i|\left(H-e_{i}\right)^{2}|j>|^{2} /\left(e_{i}-e_{j}\right) \\
& +\left[\left(e_{i} / \Delta e+2\right) / \Delta e^{2}\right] \sum_{n \neq i}^{M}\left[1 /\left(e_{i}-e_{n}\right)-1 / \Delta e\right]|<i|\left(H-e_{i}\right)^{2}|n>|^{2} \\
& \left.+(1 / \Delta e)^{3} \sum_{n \neq i}^{M}\left[1 /\left(e_{i}-e_{n}\right)-1 / \Delta e\right]<i\left|\left(H-e_{i}\right)^{3}\right| n\right\rangle\left\langle n\left|\left(H-e_{i}\right)^{2}\right| i\right\rangle \\
& +(1 / \Delta e)^{2} \sum_{n \neq 1}^{M}\left[1 /\left(e_{i}-e_{n}\right)-1 / \Delta e\right]^{2}\left(e_{n}-e_{i}\right)|<i|\left(H-e_{i}\right)^{2}|n>|^{2} \\
& +\left(e_{i} / \Delta e^{2}\right) \sum_{n \neq i}^{M}\left[1 /\left(e_{i}-e_{n}\right)-1 / \Delta e\right]^{2}|<i|\left(H-e_{i}\right)^{2}|n>|^{2} \\
& -(1 / \Delta e)^{2} \sum_{j=0}^{i-1} e_{j} /\left(e_{i}-e_{j}\right)\left[1 /\left(e_{i}-e_{j}\right)-1 / \Delta e\right]\left|\left\langle j\left|\left(H-e_{i}\right)^{2}\right| i\right\rangle\right|^{2}
\end{aligned}
$$

Regrouping terms in equation (B.3a) according to powers of $\Delta e$ and making appropriate cancellations yields 


$$
\begin{aligned}
& \left.<\psi_{\mathrm{T}}\left|\mathrm{H}^{\star}\right| \psi_{\mathrm{T}}\right\rangle \\
& =(1 / \Delta e)^{4}\left\{-e_{i}<i\left|\left(H-e_{i}\right)^{2}\right| i\right\rangle^{2}-2<i\left|\left(H-e_{i}\right)^{2}\right| i><i\left|\left(H-e_{i}\right)^{3}\right| i> \\
& +e_{i}\left\langle i\left|\left(H-e_{i}\right)^{4}\right| i>+\left\langle i\left|\left(H-e_{i}\right)^{5}\right| i>\right.\right. \\
& -2 \sum_{n \neq i}^{M}\left\langle i\left|\left(H-e_{i}\right)^{3}\right| n\right\rangle\left\langle n\left|\left(H-e_{i}\right)^{2}\right| i\right\rangle \\
& \left.\left.\sum_{n \neq i}^{M}|<i|\left(H-e_{i}\right)^{2}|n\rangle\right|^{2}\left(e_{n}-2 e_{i}\right)\right\} \\
& +(1 / \Delta e)^{3}\left\{-4<i\left|\left(H-e_{i}\right)^{2}\right| i>+4 e_{i}\left\langle i\left|\left(H-e_{i}\right)^{3}\right| i\right\rangle\right. \\
& +4<i\left|\left(H-e_{i}\right)^{4}\right| i>+\sum_{n \neq i}^{M}\left[-2|<i|\left(H-e_{i}\right)^{2}|n>|^{2}+2<i\left|\left(H-e_{i}\right)^{3}\right| n\right\rangle \\
& \left.\left.x<n\left|\left(H-e_{i}\right)^{2}\right| i>/\left(e_{i}-e_{n}\right)\right]\right\} \\
& +(1 / \Delta e)^{2}\left\{4 e_{i}<i\left|\left(H-e_{i}\right)^{2}\right| i>+6<i\left|\left(H-e_{i}\right)^{3}\right| i>\right. \\
& +\sum_{n \neq i}^{M}\left[3 /\left(e_{i}-e_{n}\right)+e_{i} /\left(e_{i}-e_{n}\right)^{2}\right]|<i|\left(H-e_{i}\right)^{2}|n>|^{2} \\
& \left.\sum_{j=0}^{i-1} e_{j}\left|\left\langle j\left|\left(H-e_{i}\right)^{2}\right| i\right\rangle\right|^{2} /\left(e_{i}-e_{j}\right)^{2}\right\}+4<i\left|\left(H-e_{i}\right)^{2}\right| i>/ \Delta e \\
& +e_{i}
\end{aligned}
$$

Similiarly the norm of the trial function is given by

$$
\begin{aligned}
& \left\langle\psi_{T}\right| \psi_{T}>=\left[1-<i\left|\left(H-e_{i}\right)^{2}\right| i>/ \Delta e^{2}\right]^{2}+\left[1-<i\left|\left(H-e_{i}\right)^{2}\right| i>/ \Delta e^{2}\right] \\
& x<i\left|\left(H-e_{i}\right)^{2}\right| i>/ \Delta e^{2}+4<i\left|\left(H-e_{i}\right)^{2}\right| i>/ \Delta e^{2}+2<i\left|\left(H-e_{i}\right)^{3}\right| i>/ \Delta e^{3} \\
& +\left[1-<i\left|\left(H-e_{i}\right)^{2}\right| i>/ \Delta e^{2}\right]<i\left|\left(H-e_{i}\right)^{2}\right| i>/ \Delta e^{2} \\
& +2<i\left|\left(H-e_{i}\right)^{3}\right| i>/ \Delta e^{3}+<i \mid\left(H-e_{i}\right)^{4} j i>/ \Delta e^{4}
\end{aligned}
$$




$$
\begin{aligned}
& +\left(2 / \Delta e^{3} \sum_{n \neq i}^{M}|<i|\left(H-e_{i}\right)^{2}|n>|^{2}\left[1 /\left(e_{i}-e_{n}\right)-1 / \Delta e\right]\right. \\
& +(1 / \Delta e)^{2} \sum_{n \neq i}^{M}|<n|\left(H-e_{i}\right)^{2}|i>|^{2}\left[1 /\left(e_{i}-e_{n}\right)-1 / \Delta e\right]^{2} \\
& =(1 / \Delta e)^{4}\left[-<i\left|\left(H-e_{i}\right)^{2}\right| i>^{2}+<i\left|\left(H-e_{i}\right)^{4}\right| i>\right. \\
& \left.-\sum_{n \neq i}^{M}|<n|\left(H-e_{i}\right)^{2}|i>|^{2}\right]+4<i\left|\left(H-e_{i}\right)^{3}\right| i>/ \Delta e^{3} \\
& +(1 / \Delta e)^{2}\left[4<i\left|\left(H-e_{i}\right)^{2}\right| i>+\sum_{n \neq i}^{M}|<n|\left(H-e_{i}\right)^{2}|i>|^{2} /\left(e_{i}-e_{n}\right)^{2}\right] \\
& +1
\end{aligned}
$$

Hence the final result for the variational energy, $W$ is

$$
\begin{aligned}
& \mathrm{W}=\left\langle\psi_{\mathrm{T}}\left|\mathrm{H}^{\star}\right| \psi_{\mathrm{T}}>/<\psi_{\mathrm{T}}\right| \psi_{\mathrm{T}}> \\
& =\left\{-e_{i}<i\left|\left(H-e_{i}\right)^{2}\right| i>-2<i\left|\left(H-e_{i}\right)^{2}\right| i>\left\langle i\left|\left(H-e_{i}\right)^{3}\right| i>\right.\right. \\
& +e_{i}<i\left|\left(H-e_{i}\right)^{4}\right| i>+<i\left|\left(H-e_{i}\right)^{5}\right| i>-2 \sum_{n \neq i}^{M}<i\left|\left(H-e_{i}\right)^{3}\right| n><n\left|\left(H-e_{i}\right)^{2}\right| i> \\
& +\sum_{n \neq i}^{M}|<i|\left(H-e_{i}\right)^{2}|n>|^{2}\left(e_{n}-2 e_{i}\right) \\
& +\left[-4<i\left|\left(H-e_{i}\right)^{2}\right| i\right\rangle^{2}+4 e_{i}\left\langle i\left|\left(H-e_{i}\right)^{3}\right| i\right\rangle \\
& +4<i\left|\left(H-e_{i}\right)^{4}\right| i>+\sum_{n \neq i}^{M}\left(-2|<i|\left(H-e_{i}\right)^{2}|n>|^{2}+2<i\left|\left(H-e_{i}\right)^{3}\right| n\right\rangle \\
& \left.\left.x<n\left|\left(H-e_{i}\right)^{2}\right| i>/\left(e_{i}-e_{n}\right)\right)\right] \Delta e+\left[4 e _ { i } \left\langlei\left|\left(H-e_{i}\right)^{2}\right| i>+6<i\left|\left(H-e_{i}\right)^{3}\right| i>\right.\right. \\
& \sum_{n \neq i}^{M}\left(3+e_{i} /\left(e_{i}-e_{n}\right)\right) /\left(e_{i}-e_{n}\right)|<i|\left(H-e_{i}\right)^{2} \cdot|n>|^{2} \\
& \left.\left.\sum_{j=0}^{i-1} e_{j}|<j|\left(H-e_{i}\right)^{2}|i>|^{2} /\left(e_{i}-e_{j}\right)^{2}\right] \Delta e^{2}+4<i\left(H-e_{i}\right)^{2} \mid i>\Delta e^{3}+e_{i} \Delta e^{4}\right\}
\end{aligned}
$$




$$
\begin{aligned}
& \quad \mid\left\{-<i\left|\left(H-e_{i}\right)^{2}\right| i\right\rangle^{2}+\left\langle i\left|\left(H-e_{i}\right)^{4}\right| i>-\sum_{n \neq i}^{M}\right|\left\langle n\left|\left(H-e_{i}\right)^{2}\right| i>\left.\right|^{2}\right. \\
& +4<i\left|\left(H-e_{i}\right)^{3}\right| i>\Delta e+\left[4<i\left|\left(H-e_{i}\right)^{2}\right| i>\right.
\end{aligned}
$$$$
\left.\left.+\sum_{n \neq i}^{M}|<n|\left(H-e_{i}\right)^{2}|i>|^{2} \Delta e /(e-e)^{2}\right] \Delta e^{2}+\Delta e^{4}\right\}
$$
$n \neq i$

In order to solve for $W$ let $\left\{a_{i}\right\}$ and $\left\{b_{i}\right\} \quad(i=0, \ldots, 4\}$ be the coefficients of numerator and denominator respectively in equation (B.5) and let $x=\Delta e . W$ is then written as

$$
w=\sum_{i=0}^{4} a_{i} x^{i} / \sum_{i=0}^{4} b_{i} x^{i}
$$

Therefore

$$
\begin{aligned}
d w / d x= & -\left\{-a_{1} b_{0}+a_{0} b_{1}+\left(-2 a_{2} b_{0}+2 a_{0} b_{2}\right) x+\left(a_{1} b_{2}+3 a_{0} b_{3}-3 a_{3} b_{0}-a_{2} b_{1}\right) x^{2}\right. \\
& +\left(2 a_{1} b_{3}+4 a_{0} b_{4}-4 a_{4} b_{0}-2 a_{3} b_{1}\right) x^{3}+\left(-a_{3} b_{2}+3 a_{1} b_{4}-3 a_{4} b_{1}+a_{2} b_{3}\right) x^{4} \\
& \left.+\left(-2 a_{4} b_{2}+2 a_{2} b_{4}\right) x^{5}+\left(-a_{4} b_{3}+a_{3} b_{4}\right) x^{6}\right\} /\left(\sum_{i=0} b_{i} x^{i}\right)^{2}
\end{aligned}
$$

The solutions of $\mathrm{dW} / \mathrm{dx}=0$ give approximate average energy $\Delta e$. 
Appendix 2: Listing of the Eigenstate Generating Program

program eisg

$\mathrm{C}$

This program can calculate eigenvalues and eigenvectors

c by the Jacobi plane rotation method

implicit double precision $(a-h, o-z)$

dimension $a(n, n), s(n, n), \operatorname{ap}(n), \operatorname{aq}(n)$

$\operatorname{read}(5, *) n,(a(i, j), j=i, n), i=1, n)$

$\mathrm{C}$

$n$ by $n$ is the size of matrix a

C

a is the matrix whose eigenvalues will be calculated $\mathrm{mm}=100000$

error $=1.0 d-6$

do $4 i=1, n$

do $4 j=i, n$

$4 a(j, i)=a(i, j)$

do $5 i=1, n-1$

$s(i, i)=1.0 \mathrm{do}$

do $5 j=i+1, n$

$s(i, j)=0.0 \mathrm{do}$

$s(j, i)=s(i, j)$

5 continue

$s(n, n)=1.0 d 0$

do $100 \mathrm{ip}=1, \mathrm{n}-1$

do $90 \mathrm{k}=1, \mathrm{~mm}$

call $\operatorname{maxv}(n, a, i p, i q, s 1)$

if (sl.le.error) go to 100

$r=(a(i q, i q)-a(i p, i p)) /(2.0 \mathrm{~d} 0 * a(i p, i q))$

$d=\operatorname{dsqrt}\left(1.0 \mathrm{d0}+r^{\star} r\right)$

$d 1=-r+d$

$d 2=-r-d$

d11=dabs $(d 1)$

$\mathrm{d} 22=\mathrm{dabs}(\mathrm{d} 2)$ 


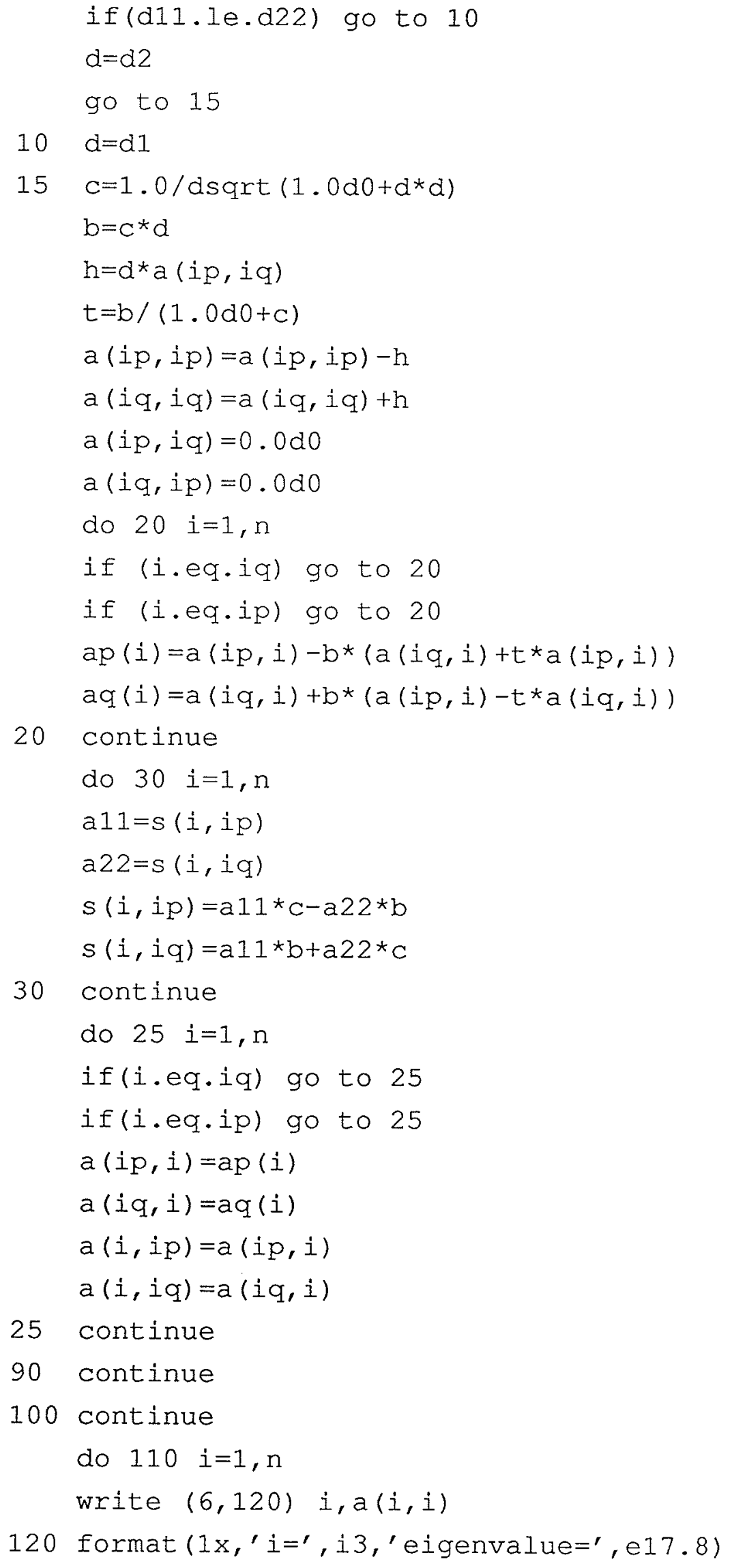




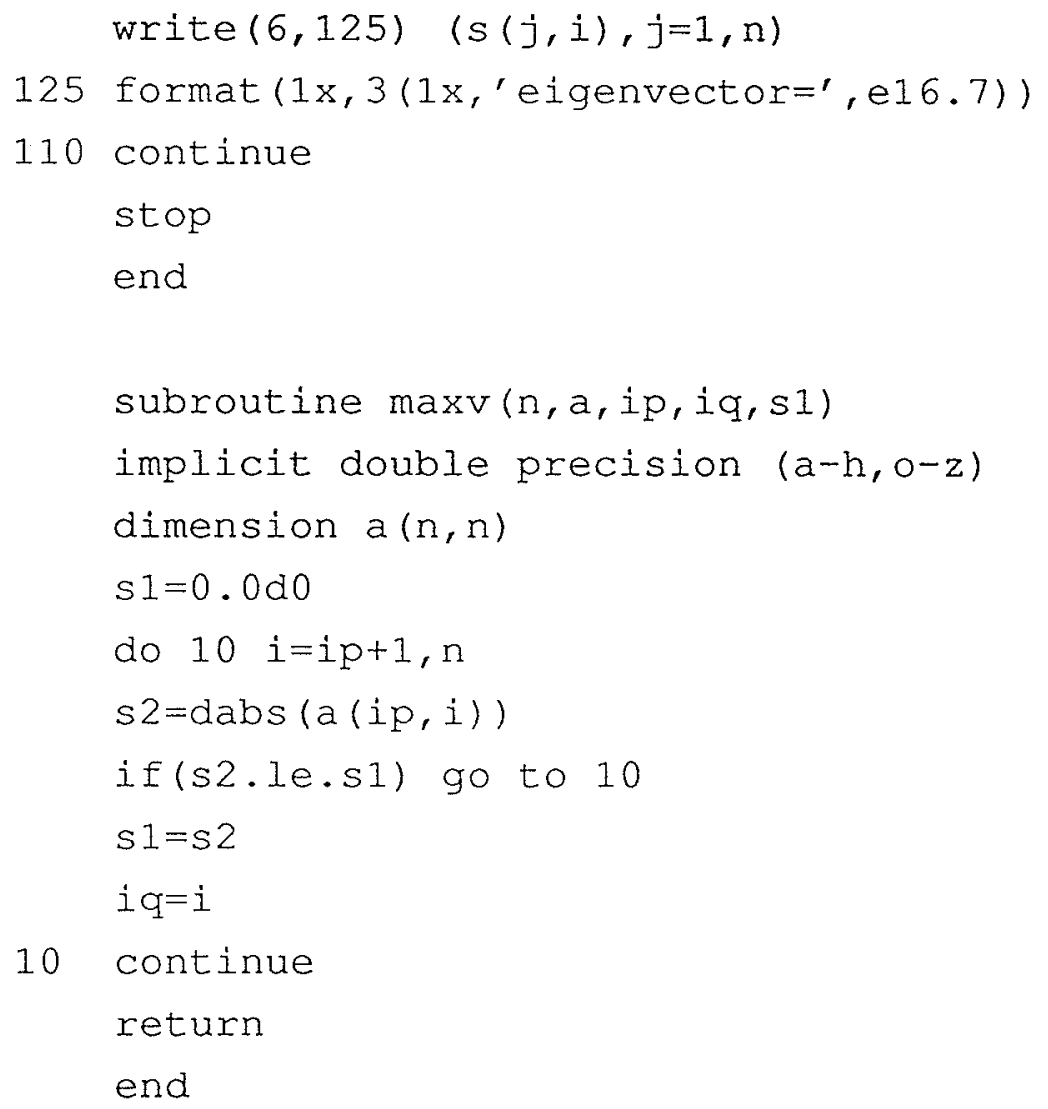




\section{Appendix 3 CDPT Program in MAPLE}

A. Introduction

The procedure for the multi-reference CDPT calculation in this thesis can be given by the following diagram

Step 1

Start

Step 2

get the optimized $r_{i}(i=0$ to 4$)$

by hop (fu, 1, m,ei) and

intt 1 ( $f u, r 0, r 1, r 2, r 3, r 4$ )

Step 3

get the matrix elements

$<g_{i}|H| g_{j}>(i, j=0$ to 4$)$ by

hop ( $f u, 1, m, e i$ ) and

intt $1(\mathrm{fu}, \mathrm{r} 0, \mathrm{r} 1, \mathrm{r} 2, \mathrm{r} 3, \mathrm{r} 4 \mathrm{~s})$

using program in Appendix 2

Step 4

to get starting point $\left\{e_{i}, \mid i>\right\}$

$\left|i>=\sum_{j=0} c_{i j}\right|^{g} j^{\prime} i=0$ to 4

$$
\begin{aligned}
& \mid \\
& \text { Let ei=e }{ }_{i}|f i>=| i>\quad(i=0 \text { to } 4) \\
& \text { save them in result.m }
\end{aligned}
$$

Step 5

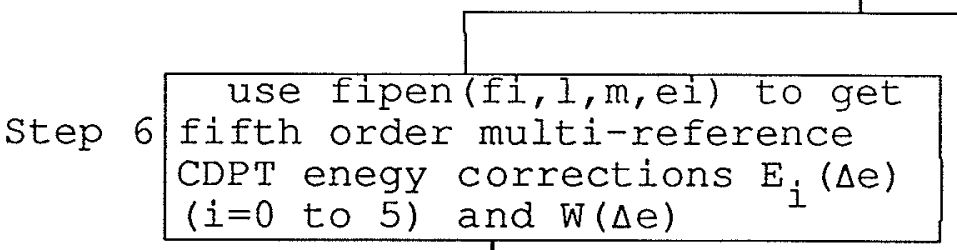

use thden( $f i, 1, m$, ei) to get third order CDPT energy corrections $E_{i}(\Delta e) \quad(i=0$ to 3$) \& W(\Delta e)$

Step 7

get the optimized $\Delta e$ and obtain
energy corrections

do double Pade calculation by the program in Appendix 3.5

End 
In this appendix those calculations in MAPLE are given by the following.

$B$ hop (fu, l, m,ei)

This subroutine is used to calculate the intermediate state after the operator, $\left[-(1 / 2) \nabla^{2}+(1 / 2) x^{2}+1 x^{3}+m x^{4}-e_{i}\right]$, acts on state $\mid \mathrm{fu}>$.

Input parameters of subroutine hop

fu function is to be acted on by operator.

1 the coefficient of cubic term of potential

$m$ the coefficient of quartic term of potential

ei starting eigenvalue of ith unperturbed state.

hop : $=\operatorname{proc}(f u, 1, m, e i)$;

ftt $:=-\operatorname{diff}($ fu, $x, x) / 2+\left(x^{\wedge} 2 / 2+1 * x^{\wedge} 3+m^{\star} x^{\wedge} 4\right) *$ fu-ei*fu;

ftt $:=$ radsimp (ftt);

ftt;

end;

save'result.m';

C.Integral Program intt 1 (fu, r0, r1, r2, r3, r4)

This integral program intt 1 uses one subroutine lmk (fu,r,itg) which uses results saved in feco.m. In the following results feco.m and subroutine lmk are given before intt 1 .

I. feco.m

This process is used to calculate integral

$$
\int_{-\infty}^{+\infty} x^{2 n} \exp \left(-r x^{2}\right)=\operatorname{co}[n] \quad(n=1, \ldots, 18)
$$

and $\quad \int_{-\infty}^{+\infty} \exp \left(-r x^{2}\right)=c 0$ 


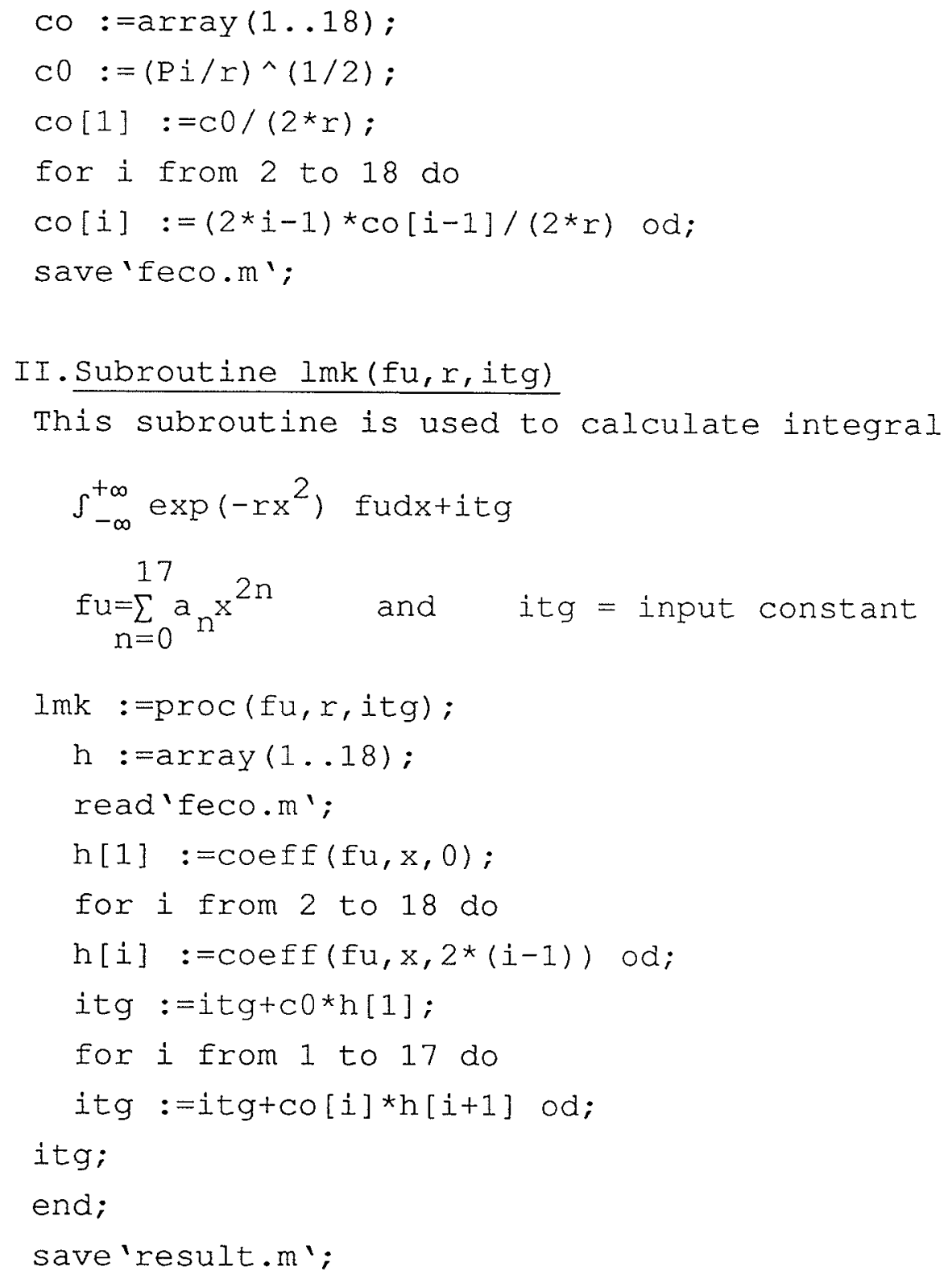

II. Integral Program intt1 ( $f u, r 0, r 1, r 2, r 3, r 4, r 5$ ) This program is used to calculate integral

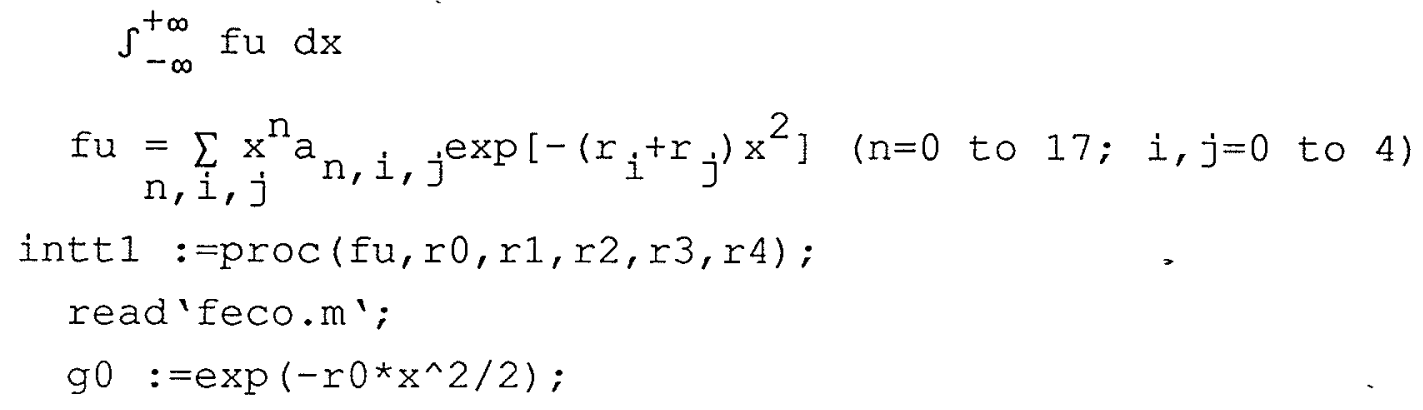




$$
\begin{aligned}
& g 1:=\exp \left(-r 1 * x^{\wedge} 2 / 2\right) \text {; } \\
& \text { g2: }=\exp \left(-r 2{ }^{\star} x^{\wedge} 2 / 2\right) \text {; } \\
& \text { g3: }=\exp \left(-r 3^{\star} x^{\wedge} 2 / 2\right) \text {; } \\
& g 4:=\exp \left(-r 4 * x^{\wedge} 2 / 2\right) \text {; } \\
& \text { ff :=expand (fu); } \\
& 10:=\operatorname{coeff}(f f, g 0,2) \text {; } \\
& \text { m0 :=coeff }(f f, g 1,2) \text {; } \\
& \text { no:=coeff }(f f, g 2,2) \text {; } \\
& j 0:=\operatorname{coeff}(f f, g 3,2) \text {; } \\
& \mathrm{k0}:=\operatorname{coeff}(\mathrm{ff}, \mathrm{g} 4,2) \text {; } \\
& 101:=\text { coeff (ff, g0,1); } \\
& \operatorname{lm}:=\text { coeff }(101,91,1) \text {; } \\
& \text { In :=coeff }(101, g 2,1) \text {; } \\
& 1 j:=\operatorname{coeff}(101,93,1) \text {; } \\
& \text { lk:=coeff }(101,94,1) \text {; } \\
& \text { m01 :=coeff }(f f, g 1,1) \text {; } \\
& m n:=\operatorname{coeff}(m 01, g 2,1) \text {; } \\
& \text { mj := coeff }(m 01, g 3,1) \text {; } \\
& \mathrm{mk}:=\operatorname{coeff}(\mathrm{m01}, \mathrm{g} 4,1) \text {; } \\
& \text { n01:= coeff (ff,g2,1); } \\
& n j:=\operatorname{coeff}(n 01, \mathrm{~g} 3,1) \text {; } \\
& \text { nk: = coeff }\left(n 01, g^{4}, 1\right) \text {; } \\
& j 01:=\operatorname{coeff}(\mathrm{ff}, \mathrm{g} 3,1) \text {; } \\
& j k:=\operatorname{coeff}(j 01,94,1) \text {; } \\
& \text { itg }:=\operatorname{lmk}(10, r 0,0) \text {; } \\
& \text { itg :=lmk (m0, r1,itg); } \\
& \text { itg }:=\operatorname{lmk}(\mathrm{n} 0, \mathrm{r} 2, \mathrm{itg}) \text {; } \\
& \text { itg }:=\operatorname{lmk}(j 0, r 3, i t g) \text {; } \\
& \text { itg :=lmk }(\mathrm{k} 0, \mathrm{r} 4, \mathrm{itg}) \text {; } \\
& r:=(r 0+r 1) / 2 \text {; } \\
& \text { itg }:=\operatorname{lmk}(\mathrm{lm}, r, i t g) \text {; } \\
& r:=(r 0+r 2) / 2 \text {; } \\
& \text { itg :=lmk (ln, r, itg); } \\
& r:=(r 0+r 3) / 2 \text {; } \\
& =\quad \text { itg : }=\operatorname{lmk}(1 j, r, i t g) \text {; } \\
& r:=(r 0+r 4) / 2 \text {; } \\
& \text { itg }:=\operatorname{lmk}(1 \mathrm{k}, \mathrm{r} \text {, itg); }
\end{aligned}
$$




$$
\begin{aligned}
& r:=(r 1+r 2) / 2 \text {; } \\
& \text { itg :=lmk (mn, } r, i t g) \text {; } \\
& r:=(r 1+r 3) / 2 \text {; } \\
& \text { itg :=lmk (mj,r,itg); } \\
& r:=(r 1+r 4) / 2 \text {; } \\
& \text { itg :=lmk (mk,r,itg); } \\
& r:=(r 2+r 3) / 2 \text {; } \\
& \text { itg := } \operatorname{lmk}(n j, r, i t g) \text {; } \\
& r:=(r 2+r 4) / 2 \text {; } \\
& \text { itg }:=\operatorname{lmk}(n k, r, i t g) ; \\
& r:=(r 3+r 4) / 2 \text {; } \\
& \text { itg : }=\operatorname{lmk}(j k, r, i t g) \text {; } \\
& \text { itg; }
\end{aligned}
$$

end;

D. The Program for Multi-reference CDPT to Eifth Order

This is the CDPT main program by which the variational energy $W$ and perturbation energy corrections of the j'th state to fifth order are calculated. In addition, the trial function $\psi_{\mathrm{T}}$ is formed by the sum of the first two wave function corrections and starting function.

Before calling the subroutine fipen, the five unperturbed states, corresponding energies, and the integral programs inttl given above as well as the subroutine hop (fu, l, m, ei) should be stored (or saved) in result.m. Note: This program can be used only for starting functions as described in Chapter 2, Section 2.4.

Input parameters of subroutine fipen:

fj $j^{\prime}$ th unperturbed state

1 the coefficient of cubic term in potential

m the coefficient of quartic term in potentiai 


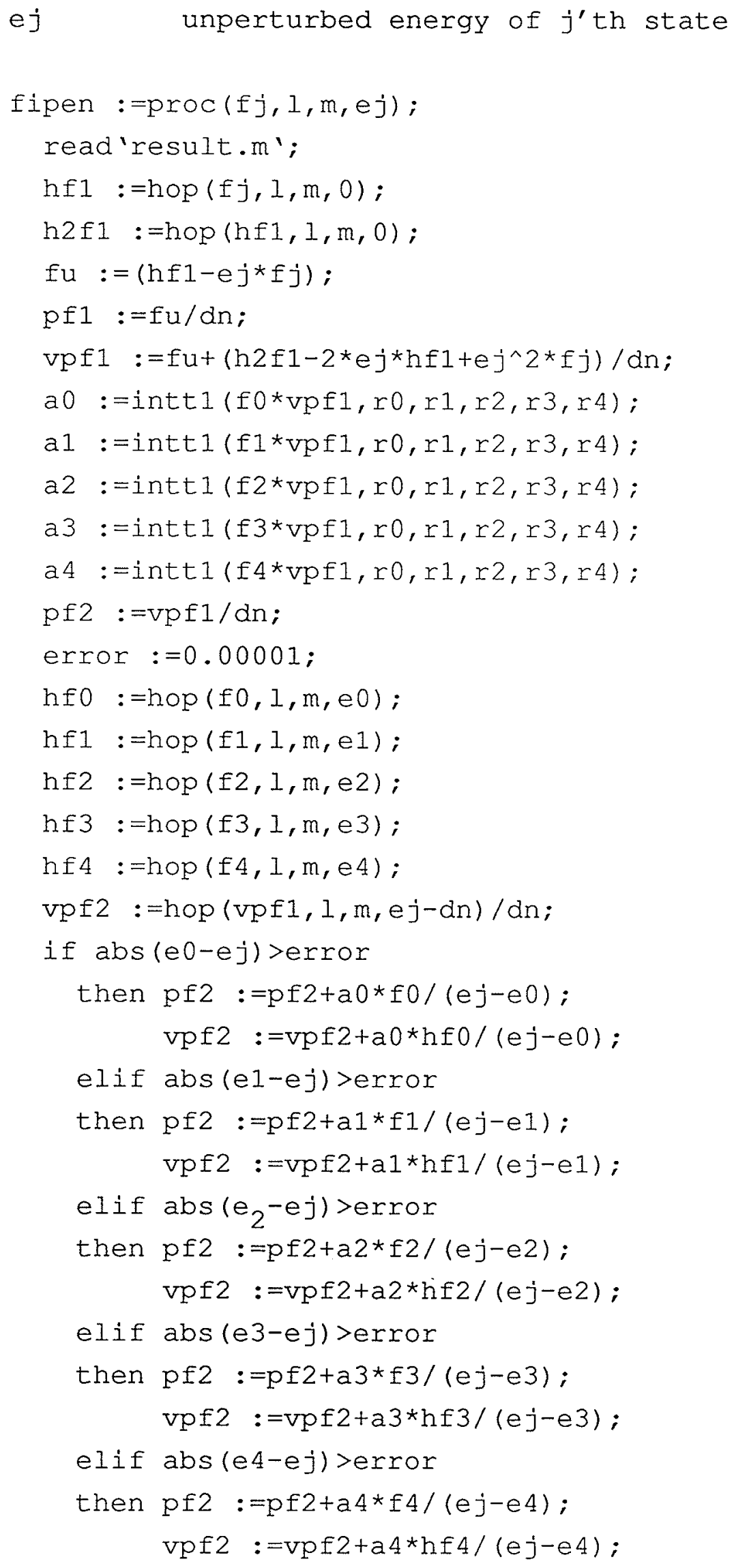


fi;

pf2 :=pf2-(a0*f0+a1*f1+a2*f2+a3*f3+a4*f4)/dn;

vpf2 :=vpf2-(a0*hf0+a1*hf1+a $2 * h f 2+a 3 * h f 3+a 4 * h f 4) / d n$;

vpf2 :=vpf2-( (ej-e0+dn)*a $0 * f 0+(e j-e 1+d n) * a 1 * f 1$

$+(e j-e 2+d n) * a 2 * f 2+(e j-e 3+d n) * a 3 * f 3$

$+(e j-e 4+d n) * a 4 * f 4) / d n$;

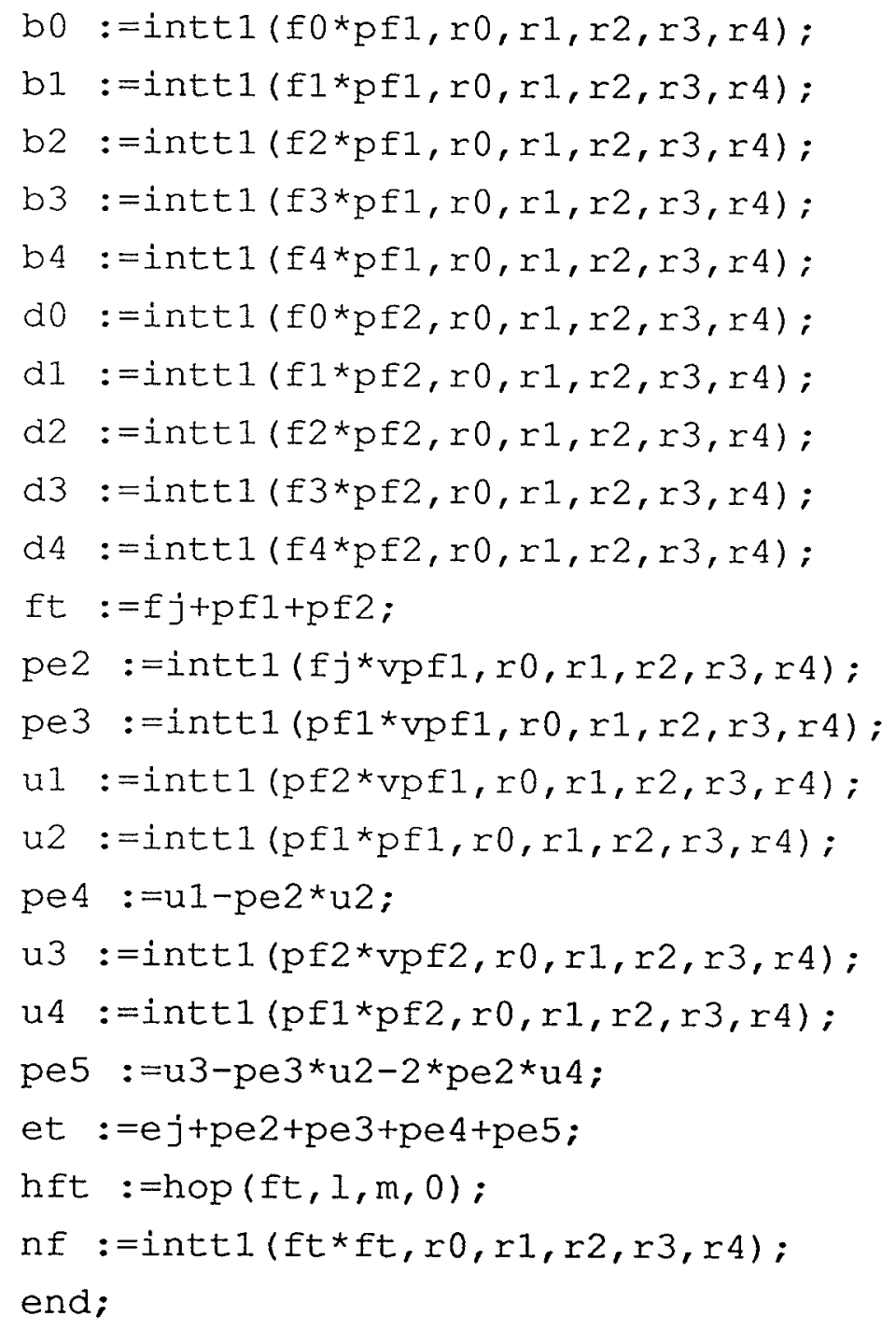

E. Double Pade Program in MAPLE

This program is used to do the double Pade calculation. e10:=e0e1+e2/(1'-e3/e2);

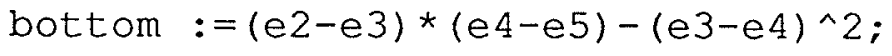
e21: $=e 0 e 1+(e 2 *(e 4-e 5)-e 3 *(e 3-e 4)) * e 2 /$ bottom 


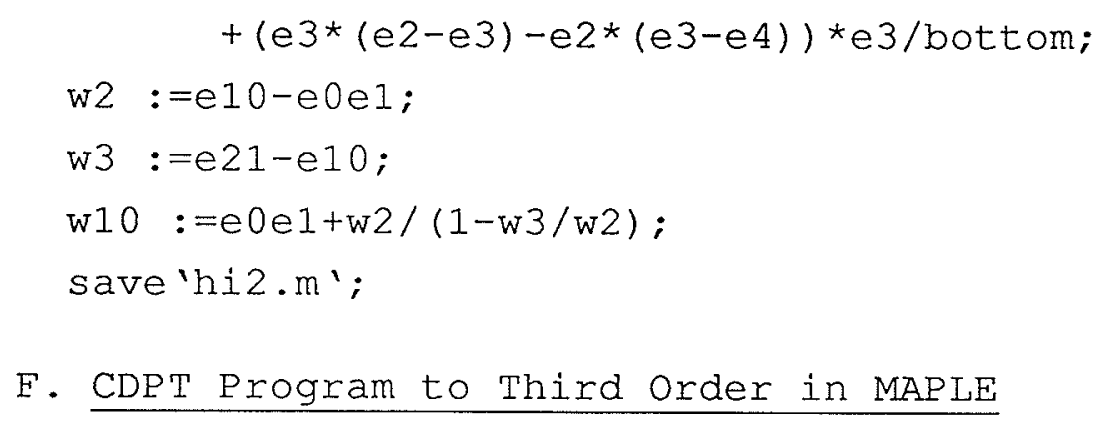

This is the CDPT program by which the variational energy and perturbation energy can be calculated to third order. The trial function $\psi_{\mathrm{T}}$ is formed by the sum of the first order wave function correction and starting function.

Before calling subroutine thden the integral programs inttl, hop, and five unperturbed states with eigenvalues should be read.

Note: Again this program can be used only for starting functions described as Chapter2, Section 2.4.

Input parameters of subroutine thden:

fj j'th unperturbed state

1 the coefficient of cubic term in potential

$m$ the coefficient of quartic term in potential

ej $\quad j^{\prime}$ th unperturbed eigenvalue

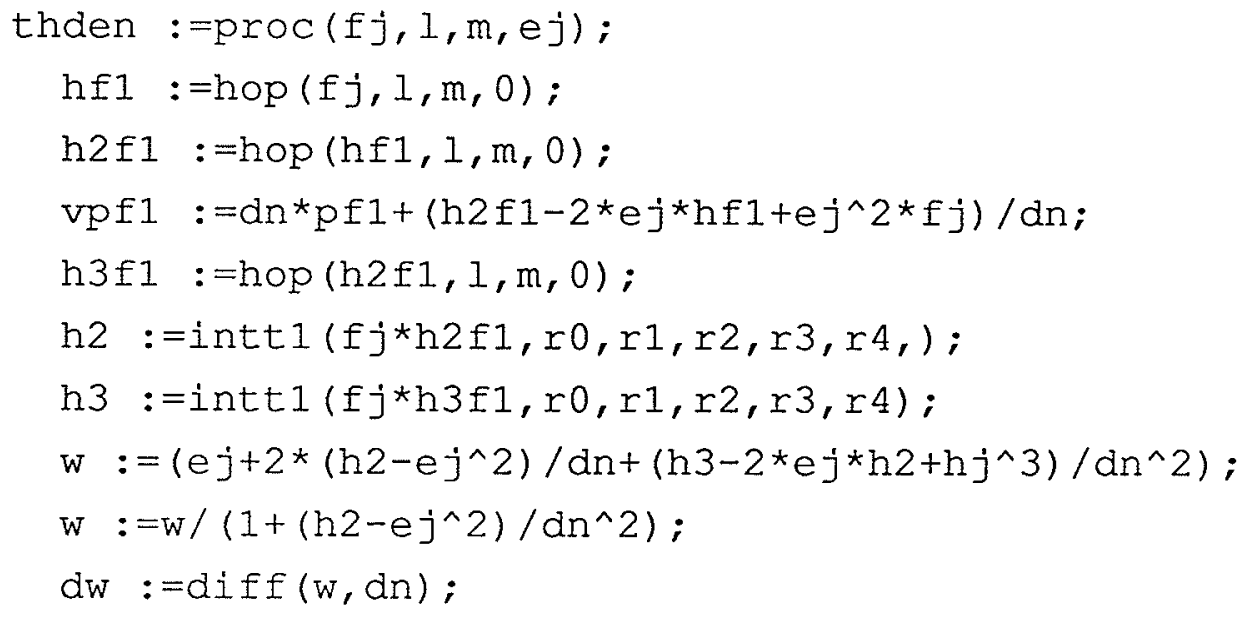




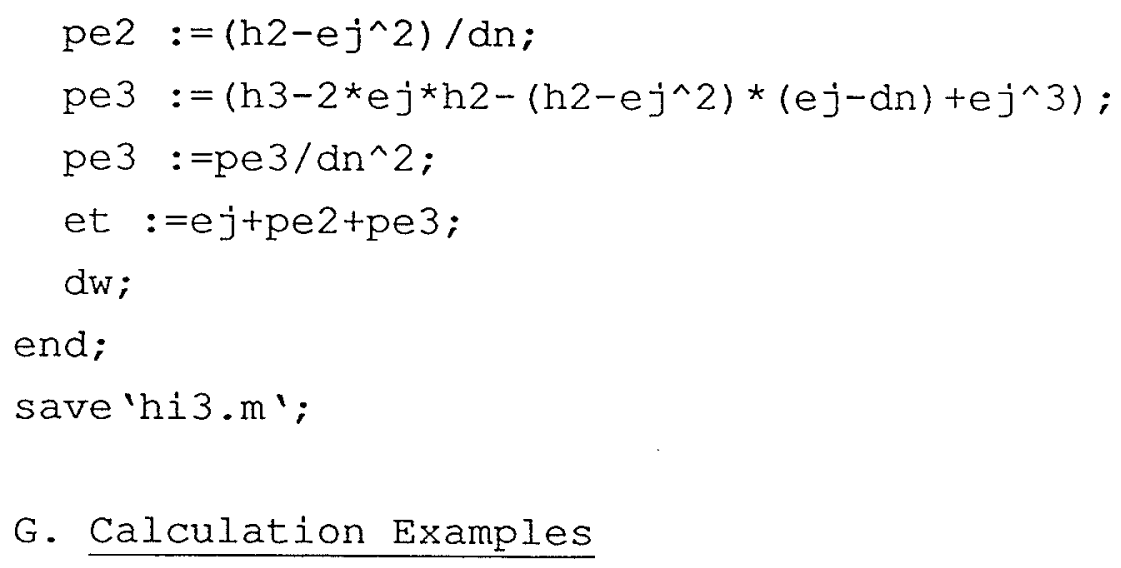

Step 4. Use program in Appendix 2 to get eigenvalues and corresponding eigenvectors $\left[\left\{e_{i}\right\}, c_{i j} i j=0, \ldots, 4\right]$.

Step 5. Define reference functions and starting point

=.g. the first reference function |0>

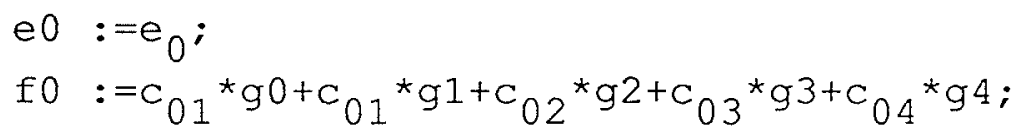


Step 6. Do CDPT perturbation calculation

e.g. do perturbation to ground state $|0\rangle$

fipen ( $f 0, I, m, e 0)$ :

$\mathrm{w}:=$ intt 1 (ft*hft, ro, r1, r2, r3, r4):

$\mathrm{w}:=\mathrm{w} / \mathrm{nf}:$

$\mathrm{dw}:=\operatorname{diff}(\mathrm{w}, \mathrm{dn}):$

solution :=solve $(d w, d n)$;

dn :=solution;

or do the third order CDPT calculation only substituting thden into fipen subroutine above.

Step 7. Pade calculation

e.g. do calculation to ground state

read'hi2.m';

e0e1 :=eo;

e2 :=pe2;

e3 :=pe3;

e4 :=pe 4;

e5 :=pe5;

e10;

e21;

w10; 


\section{References:}

1. Warren J. Hehre; Ab Initio Molecular Orbital Theory (Wiley, New York, 1986);p283

2. B. G. Wicke and D. O. Harris; J. Chem. Phys.; 64, $5236(1976)$

3. R. I. Somorjai and D. F. Hornig; J. Chem. Phys.; 36, $1980(1962)$

4. B. R. Johnson; J. Chem. Phys.; 67, 4086 (1977)

5. J. P. Leroy and R. Wallace; J. Phys. Chem.; 89, 1928 (1985)

6. E. A. MCCullough J.; Comp. Phys. Reports; 4, 265 (1986)

7. C. Froese Fisher; The Hartree Fock Method for Atoms (Wiley, New York, 1977)

8. J. Navaza; Phys. Rev. A; 24, 683(1981)

9. H. J. Monkhorst and B. Jeriorski; J. Chem. Phys.; 71, $5268(1979)$

10.W. L. McMillan; Phys. Rev. A; 138,442(1965)

11. D. M. Ceperley; G. V. Chester and M. H. Kalos; Phys. Rev. B; 16, 3081 (1977).

12. J. W. Moskowitz and M. H. Kalos; Int. J. Quantum Chem.; $20,1107(1981)$

13. J. B. Anderson; J. Chem. Phys.; 73,3897 (1982)

14. D. M. Ceperley and B. J. Alder; Phys. Rev. Lett.; 45, $566(1980)$

15. P. J. Reynolds; J. Chem. Phys.; 77,5593 (1982)

16. A. Unsöld; Z. Physik; 43, 563 (1927)

17. M. G. Sylvain; Chem. Phys. Lett.; 86, 1632 (1987)

18. P. W. Fowler; Molec. Phys.; 53, 865 (1984) 
19. P. W. Fowler; Molec. Phys.; 54, 129(1985)

20. D. Vanlabeke; J. Chem. Phys.; 86, 1632 (1987)

21. P. Piecuch; Molec. Phys.; 59, 1085 (1986)

22. P. Piecuch; Molec. Phys.; 59, 1097 (1986)

23. J. O. Hirschfelder and W. Byers Brown; Adv. Quantum Chem.; $1,256(1964)$

24. Review; Ad van der Avoird et al.; Topics in Current Chem.; $93,1(1980)$

25. E. Mulder et al.; Theoret. Chim. Acta; 46, 39(1977)

26. H. N. W. Lekkerkerker et al.; Physica; 88A, 375 (1977)

27. R. Luyckx et al. J. Chem. Phys.; 70, 4212 (1979)

28. J. L. Rivail and A.Cartier; Mol. Phys.; 36, 1085 (1978)

29. John M. Cullen and Michael C. Zerner; Theoret. Chim. Acta; $61,203(1982)$

30. E. A. Hylleraas, Z. Phys.; 65, 209(1930)

31. Bernard Kirtman and M. L. Benston; J. Chem. Phys.; $46,472(1967)$

32. C. Kittel; Introduction to Solid State Physics, (Wiley, New York, 1976)

33. Alan D. Isaacson. J. Chem. Phys.; 80, 2888 (1984)

34. B. J. MCCoy; J. Chem. Phys.; 80, 3629(1984)

35. R. K. Pathria; Statistical Mechanics, (Pergamon, 1972)

36. M. Quack and E. Sutcliffe; J. Chem. Phys.; 83,3805 (1985)

37. J. Chang and R. E. Wyatt; Chem. Phys. Lett.; 121, 307 (1985) 
38. J. Diaz Bejarano and A. Martin Sánchez; J. Chem. Phys. 85, $5128(1986)$

39. C. S. Hsue and J. L. Chern; Phys. Rev. D; 29, 643(1984)

40. K. Banerjee and S. P. Bhatangar; Phys. Rev. D; 18, $4767(1978)$

41. S. N. Biswas; J. Math. Phys.; 14, 1190 (1973)

42. C. M. Bender and T. T. Wu; Phys. Rev.; 184, 1231 (1969)

43. Pravat Kumar Patnaik; Phys. Rev. D; 35,1234 (1987)

44. I. G. Halliday and P. Suranyi; Phys. Rev. D; 21, 1529(1980)

45. J. Killingbeck; Phys. Lett. 65A, 87 (1978)

46. J. Killingbeck; J. Phys. A; 19, 705(1986)

47. C. S. Lai; Phys. Rev. A; 23, 455 (1981)

48. C. S. Lai and M. P. Madan; Mol. Phys.; 54,699(1985)

49. John M. Cullen; Int. J. Quantum Chem. (in press) (1989)

50. B. W. Char, K. O. Geddes; Maple Reference Manual (Watcom Publications, Waterloo, Ont., Canada) (1985). 